\title{
Role of the reaction-structure coupling in temperature compensation of the KaiABC circadian rhythm
}

\author{
Masaki Sasai ${ }^{1, *}$ \\ ${ }^{1}$ Department of Applied Physics, Nagoya University, Nagoya, 464-8603, Japan \\ *masakisasai@nagoya-u.jp
}

\begin{abstract}
When the mixture solution of cyanobacterial proteins, KaiA, KaiB, and KaiC, is incubated with ATP in vitro, the phosphorylation level of KaiC shows stable oscillations with the temperature-compensated circadian period. We analyzed this temperature compensation by developing a theoretical model describing the feedback relations among reactions and structural transitions in the KaiC molecule. The model showed that the reduced structural cooperativity should weaken the negative feedback coupling among reactions and structural transitions, which enlarges the oscillation amplitude and period, explaining the observed significant period extension upon single amino-acid residue substitution. We propose that an increase in thermal fluctuations similarly attenuates the reaction-structure feedback, explaining the temperature compensation in the KaiABC clock. The model suggests that the ATPase reactions in the $\mathrm{Cl}$ domain of KaiC affect the period depending on how the reaction rates are modulated. The KaiABC clock provides a unique opportunity to analyze how the reaction-structure coupling regulates the system-level synchronized oscillations of molecules.
\end{abstract}

The mixture solution of cyanobacterial proteins, KaiA, KaiB, and KaiC, shows the robust structural and chemical oscillations with the period of approximately $24 \mathrm{~h}$ when the solution is incubated with ATP in vitro ${ }^{1-3}$. This period is insensitive to the temperature change, showing the feature of temperature compensation ${ }^{1}$. An important question is whether this feature has a common molecular mechanism or the same mathematical principle as temperature compensation in generic transcriptiontranslation oscillations (TTO). Circadian clocks in many organisms are driven by the time-delayed negative feedback in the $\mathrm{TTO}^{4,5}$, whose oscillation period is temperature compensated ${ }^{6,7}$; the ratio of the period in $10^{\circ} \mathrm{C}$ difference is $0.9 \lesssim Q_{10} \lesssim 1.1$, which is much closer to 1 than the value $\gtrsim 1.5$ expected from the temperature dependence of normal biochemical reactions. This temperature compensation has been studied with various theoretical models ${ }^{8-19}$, but its mechanism remains a challenging problem.

There have been at least three views or approaches to temperature compensation; (i) the balance between opposing reactions, (ii) the correlation between oscillation period and amplitude, and (iii) the role of a critical reaction step. One view is the balance between reactions opposingly working to shorten or lengthen the period upon temperature change ${ }^{7,9}$. Such balancing was mathematically formulated ${ }^{20}$ and studied with different models ${ }^{10-15}$. In searching for the balancing reactions, various mechanisms were examined, including the balance between negative and positive feedback regulations ${ }^{9}$ and the balance between ways of resetting bifurcations ${ }^{12}$. In particular, Lakin-Thomas et al. emphasized that the period length should correlate with the amplitude of oscillations ${ }^{8}$, suggesting the possible use of the correlation as a clue to find the reactions responsible for the balance ${ }^{17}$. An alternative approach is to search for the critical reaction step or the molecule that determines temperature compensation. In mammals, phosphorylation of period 2 (PER2) regulated by casein kinase $\mathrm{I} \varepsilon / \delta(\mathrm{CKI} \varepsilon / \delta$ ) is temperature insensitive $^{21}$, and this insensitivity was attributed to the reaction mechanism of the CKI $\varepsilon / \delta$ molecule $^{22}$.

Therefore, it is meaningful to analyze the temperature compensation of the KaiABC post-transcription oscillations from the three views discussed for TTO. Previously, two hypotheses on the KaiABC temperature compensation were proposed based on the view (iii) of the critical reaction step and the view (i) on the balance between competing reactions. A critical reaction step is ATP hydrolysis in KaiC. KaiC hexamer is a slow ATPase, and variation of the ATPase activity among KaiC mutants is correlated with the variation of the oscillation frequency of those mutants ${ }^{23,24}$. Because the ATPase activity of KaiC is temperature insensitive ${ }^{23}$, this correlation suggested that ATPase activity determines temperature compensation in the KaiABC oscillations. In order to clarify whether such causality exists behind the observed correlation, further experimental and theoretical investigations are necessary. Another hypothesis was based on the assumption of balance among the competing binding reactions of KaiA to KaiC at different phosphorylation levels ${ }^{25}$. With this hypothesis, the population of KaiC at a highly phosphorylated state dominates in low temperature, which increases the free unbound KaiA molecules, and increases the 
overall binding rate of KaiA to compensate for the decrease of reaction rates at the low temperature. However, the accumulation of KaiC at a highly phosphorylated state in low temperature was not observed experimentally ${ }^{1}$; therefore, exploration for other hypotheses and comparison among them are necessary.

Here, we propose a hypothesis based on the view (ii) of the correlation between oscillation period and amplitude. A recent experimental report revealed that substituting an amino-acid residue near the CI and CII domains interface in KaiC induces a striking change of the period from $15 \mathrm{~h}$ to $158 \mathrm{~h}$ with a clear correlation between period and amplitude ${ }^{26}$. The modified period length in the mutant was anti-correlated with the volume of the residue after the substitution ${ }^{26}$, indicating that the structural coupling between the CI and CII domains is crucial to determine period and amplitude; the period and amplitude were enlarged when the structural coupling between domains was weaker with the smaller volume of residue at the domain interface. The recent X-ray analysis showed that KaiC undergoes structural transitions depending on the state of two phosphorylation sites in the $\mathrm{CII}$ and the nucleotide-binding state in the $\mathrm{CI}^{27}$. This observation indicated that the structural transitions of KaiC take place through the allosteric communication between CI and CII domains depending on the phosphorylation reactions in the CII and the ATPase reactions in the CI. On the other hand, the rates of those reactions should depend on the structure. Therefore, it is plausible to assume the feedback relations among reactions and structural transitions. Substituting smaller volume residue at the CI-CII interface should reduce the transition cooperativity and weaken the feedback coupling. The weakening of the negative feedback enlarges the amplitude and lengthens the period in general nonlinear oscillators as found in the TTO model ${ }^{9}$; therefore, the observed change in the mutants can be explained if the coupling among reactions and structural transitions of KaiC constitutes the negative feedback relation.

In the present study, we propose that the structural coupling between the $\mathrm{CI}$ and $\mathrm{CII}$ is weakened through thermal fluctuations, weakening the negative feedback relations. In the higher temperature, the larger thermal fluctuations at the CI-CII interface should obscure the specific atomic interactions at the interface, producing a similar effect to the substitution to the smaller volume residue. This weakening of interactions at the interface reduces the negative feedback strength, enhancing the oscillation amplitude, lengthening the period, and compensating for the thermal acceleration of reactions in the higher temperature. We analyze this hypothesis with a model of the KaiABC oscillator and discuss possible tests of the model prediction. We also analyze the correlation between the ATPase reactions and the oscillation frequency to discuss the role of the ATPase reactions in the Kai system temperature compensation.

\section{Model}

\section{Problems at two levels; the molecular and ensemble levels}

In modeling the KaiABC oscillator, we need to analyze two mechanisms: how individual KaiC molecules oscillate and how oscillations of many KaiC molecules synchronize to generate the ensemble-level oscillations in solution. Many theoretical works focused on the latter question as the sequential change of phosphorylation level of individual KaiC molecules was assumed in advance; then, the synchronization was explained using various assumptions ${ }^{25,28-39}$.

A plausible assumption is KaiA sequestration ${ }^{25,34-44}$; the preferential KaiA binding to particular KaiC states sequestrates KaiA, reducing the KaiA binding rate in the other KaiC states, leading to the accumulation of the population in those states, and producing coherent synchronizaed oscillations. This hypothesis is consistent with the experimental observation that the ensemble oscillations disappear when KaiA is too abundant in the solution ${ }^{45}$. Various states of KaiC were assumed as the KaiA-sequestrating states; some models assumed KaiA is sequestrated into the lowly phosphorylated KaiC in the phosphorylation (P) process ${ }^{25,34-36,40}$ or in the dephosphorylation (dP) process $^{37,38}$. The other models assumed that KaiA is sequestrated by forming the KaiC-KaiB-KaiA complexes that appear during the $\mathrm{dP}$ process $\mathrm{s}^{39,41-44}$. The present author showed $^{44}$ that the assumption of the KaiA sequestration into the KaiC-KaiB-KaiA complexes quantitatively explains the experimental data on how the oscillations are entrained when two solutions oscillating at different phases are mixed ${ }^{46}$.

In the present study, we use the hypotheses of the KaiA sequestration into the KaiC-KaiB-KaiA complexes to explain the synchronization. We also model the mechanism of how oscillations are driven in individual KaiC molecules. In this way, we address the questions extending over the two levels, the individual molecular level and the ensemble level, to analyze how the molecular-level feedback coupling determines the ensemble-level oscillations and temperature compensation.

\section{Feedback coupling of reactions and structural transitions at the molecular level}

At the molecular level, KaiC forms a hexamer ${ }^{47-49}$, which is denoted here by $\mathrm{C}_{6}$. The KaiC monomer is composed of the N-terminal (CI) and C-terminal (CII) domains ${ }^{50}$, which are assembled to the CI and CII rings in $\mathrm{C}_{6}{ }^{49}$ (Fig. 1). The $\mathrm{NMR}^{51,52}$, small-angle X-ray diffraction ${ }^{53}$, biochemical analyses ${ }^{54}$, and X-ray crystallography ${ }^{27}$ showed the cooperative structural transitions of KaiC hexamer between two, the structure in the P phase and the structure in the $\mathrm{dP}$ phase. We use the order parameter $0 \leq X \leq 1$ to describe the transitions between two typical conformations and thermal fluctuations around each conformation. We write $X(k, t) \approx 1$ when the $k$ th KaiC hexamer at time $t$ takes the structure in the $\mathrm{P}$ phase, and $X(k, t) \approx 0$ when it takes the structure in the $\mathrm{dP}$ phase 


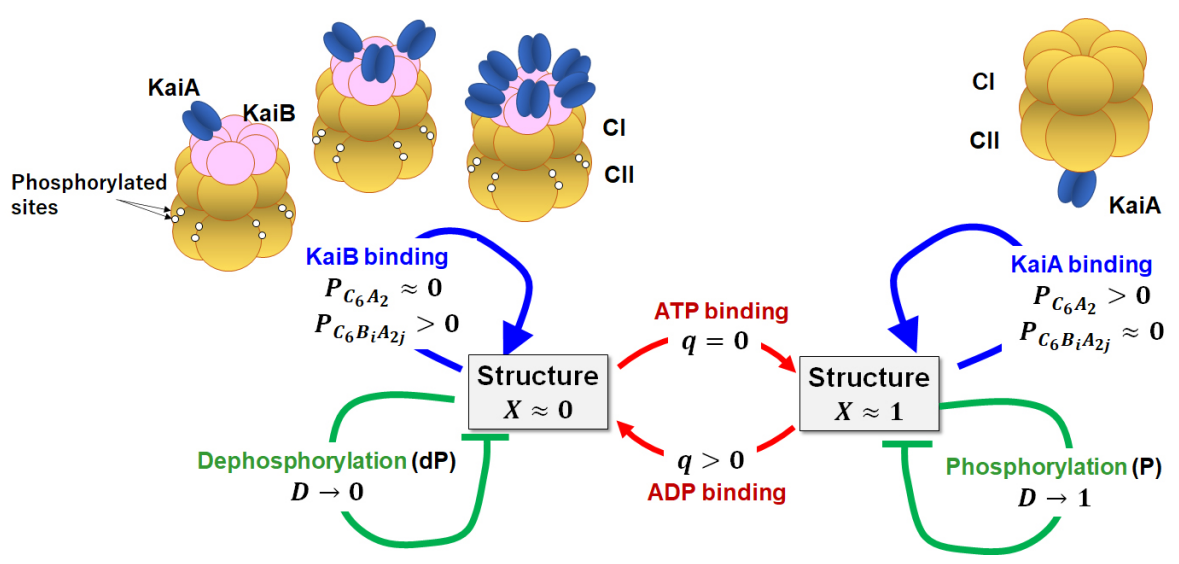

Figure 1. Feedback coupling among reactions and structural transitions in KaiC hexamer. The model is based on the fundamental experimental observations: KaiC forms a hexamer composed of the $\mathrm{CI}$ and $\mathrm{CII}$ rings. The CII has twelve sites to be phosphorylated. The KaiC hexamer undergoes allosteric transitions between two structures; the structure $(X \approx 1)$ in the phosphorylation $(\mathrm{P})$ phase and the structure $(X \approx 0)$ in the dephosphorylation $(\mathrm{dP})$ phase. A KaiA dimer can bind on the CII of the $X \approx 1 \mathrm{KaiC}$ with the probability $P_{\mathrm{C}_{6} \mathrm{~A}_{2}}$, which promotes the $\mathrm{P}$ process to increase the phosphorylation level $D$. KaiB monomers can bind on the $\mathrm{CI}$ of the $X \approx 0 \mathrm{KaiC}$, and a KaiA dimer can further bind on each KaiB monomer forming

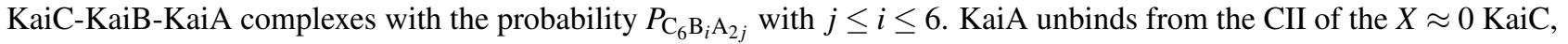
promoting the $\mathrm{dP}$ process to decrease $D$. Based on these observations, the model describes the feedback coupling in the KaiC hexamer by assuming (i) the KaiA binding on the CII stabilizes the $X \approx 1$ state, and (ii) the KaiB binding on the CI stabilizes the $X \approx 0$ structure. The assumptions (i) and (ii) account for the positive feedback to stabilize the $X \approx 1$ and $X \approx 0$ states. The model also assumes that (iii) the gradual rise of $D$ destabilizes the $X \approx 1$ structure and (iv) the gradual fall of $D$ destabilizes the $X \approx 0$ structure. The assumptions (iii) and (iv) support the time-delayed negative feedback to drive the transitions between the $X \approx 1$ and $X \approx 0$ states. The model further assumes (v) the stochastic ATP hydrolysis in the CI $(q \rightarrow 1)$ destabilizes the $X \approx 1$ state, and (vi) the stochastic ADP release from the CI and the subsequent ATP binding $(q \rightarrow 0)$ destabilize the $X \approx 0$ state. (v) and (vi) trigger the transitions between the $X \approx 1$ and $X \approx 0$ states. The assumptions (i) through (vi) generate the oscillations of individual KaiC hexamers. We hypothesized (vii) oscillations of multiple KaiC hexamers are coupled through the KaiA sequestration into the KaiC-KaiB-KaiA complexes, giving rise to the ensemble-level oscillations.

In the present model, we consider that the structural state is a hub of multifold feedback relations among reactions and structural transitions (Fig. 1). We describe individual KaiC molecules with the structural parameter $X$ and coarse-grained variables representing three types of reactions; (1) the binding/unbinding reactions of KaiA and KaiB to/from KaiC, (2) the $\mathrm{P} / \mathrm{dP}$ reactions in the CII, and (3) the ATPase reactions in the CI. We consider that these three types of reactions directly or indirectly depend on $X$, and these reactions affect $X$, constituting the multifold feedback relations.

\section{Binding/unbinding reactions of KaiA and KaiB}

$\mathrm{KaiA}$ and $\mathrm{KaiB}$ bind/unbind to/from $\mathrm{KaiC}$ in a coordinated way ${ }^{55-60}$. The CII ring of a KaiC hexamer can bind a KaiA dimer during the $\mathrm{P}$ process to form $\mathrm{C}_{6} \mathrm{~A}_{2}{ }^{36,61}$. The cryo-electron microscopy and mass-spectrometry showed that each $\mathrm{CI}$ domain can bind a KaiB monomer to form KaiC-KaiB complexes, further binding KaiA dimers to form KaiC-KaiB-KaiA complexes, $\mathrm{C}_{6} \mathrm{~B}_{i} \mathrm{~A}_{2 j}$ with $j \leq i \leq 6^{60}$. The stoichiometry $\mathrm{C}_{6} \mathrm{~B}_{i} \mathrm{~A}_{2 j}$ implies the large capacity of KaiC-KaiB-KaiA complexes to absorb KaiA molecules. We consider the probability of the $k$ th KaiC hexamer forming $\mathrm{C}_{6} \mathrm{~A}_{2}$ at time $t, P_{\mathrm{C}_{6} \mathrm{~A}_{2}}(k, t)$, and the probability forming $\mathrm{C}_{6} \mathrm{~B}_{i} \mathrm{~A}_{2 j}, P_{\mathrm{C}_{6} \mathrm{~B}_{i} \mathrm{~A}_{2 j}}(k, t)$.

Binding/unbinding of KaiA to/from the CII has a timescale of seconds ${ }^{36}$. Because this kinetics is much faster than the other reactions in KaiC, we describe $P_{\mathrm{C}_{6} \mathrm{~A}_{2}}$ with the quasi-equilibrium approximation, $P_{\mathrm{C}_{6} \mathrm{~A}_{2}}(k, t)=\frac{x_{\mathrm{A}}(t) h_{\mathrm{A}}(k, t)}{f_{\mathrm{A}}(k, t)} P_{\mathrm{C}_{6} \mathrm{~B}_{0} \mathrm{~A}_{0}}(k, t)$, where $x_{\mathrm{A}}(t)$ is the concentration of free unbound KaiA dimer at time $t . h_{\mathrm{A}}(k, t)$ and $f_{\mathrm{A}}(k, t)$ are the binding and unbinding rate constants of a KaiA dimer to and from the CII, respectively. We represent the preferential KaiA binding to the $X \approx 1$ structure by assuming that $h_{\mathrm{A}}(k, t)$ is an increasing function and $f_{\mathrm{A}}(k, t)$ is a decreasing function of $X(k, t)$ (See the Methods section).

Binding/unbinding of KaiB has a timescale of an hour ${ }^{36,39}$. We describe the slow temporal variation of $P_{\mathrm{C}_{6} \mathrm{~B}_{i} \mathrm{~A}_{2 j}}(k, t)$ by integrating the kinetic equations (Methods), which are represented with the rate constants of KaiB binding and unbinding, $h_{\mathrm{B}}(k, t)$ and $f_{\mathrm{B}}(k, t)$, respectively, and the rate constants of KaiA binding and unbinding to and from the KaiB, $h_{\mathrm{AB}}$ and $f_{\mathrm{AB}}$, respectively. We represent the tendency of preferential binding of KaiB to the $X \approx 0$ structure by assuming that $h_{\mathrm{B}}$ is a 
decreasing function and $f_{\mathrm{B}}$ is an increasing function of $X(k, t)$. Because KaiA does not directly interact with KaiC in this process, we assume $h_{\mathrm{AB}}$ and $f_{\mathrm{AB}}$ are independent of $X$. The unusually slow yet specific binding kinetics of the KaiB should be attributed to the fold transitions of $\mathrm{KaiB}$. KaiB switches between ground state $\left(\mathrm{KaiB}_{\mathrm{gs}}\right)$ and fold-switched state $\left(\mathrm{KaiB}_{\mathrm{fs}}\right)$ by changing its secondary structures ${ }^{62}$. When only $\mathrm{KaiB}_{\mathrm{fs}}$ has a significant binding affinity to the $\mathrm{CI}$ and $\mathrm{KaiB}_{\mathrm{fs}}$ is the excited state with the energy rise of $\Delta E_{\mathrm{fs}-\mathrm{gs}}>0$, the small factor $\exp \left(-\Delta E_{\mathrm{fs}-\mathrm{gs}} /\left(k_{\mathrm{B}} T\right)\right)$ explains the small $h_{\mathrm{B}}$.

\section{$P / d P$ reactions}

Each CII domain has two sites, Ser431 and Thr432, to be phosphorylated, which amounts to 12 sites in a KaiC hexamer. For simplicity, we do not distinguish Ser431 and Thr432 in the present expression, describing the phosphorylation level of 12 sites with the parameter $0 \leq D(k, t) \leq 1 ; D(k, t)=1$ when 12 sites in the CII of the $k$ th KaiC hexamer are all phosphorylated, and $D(k, t)=0$ when they are all unphosphorylated. Phosphorylation is promoted when KaiA binds on the CII ${ }^{39,55}$, and dephosporylation proceeds when it unbinds ${ }^{39}$. We represent this tendency by writing

$$
\frac{d}{d t} D(k, t)=k_{\mathrm{p}} H^{+}(k, t)[1-D(k, t)]-k_{\mathrm{dp}} H^{-}(k, t) D(k, t),
$$

where $H^{+}(k, t)=z /(1+z)$ and $H^{-}(k, t)=1 /(1+z)$ represent the effects of binding and unbinding of KaiA with $z=$ $P_{\mathrm{C}_{6} \mathrm{~A}_{2}}(k, t) / P_{0}$ and a constant $P_{0}$. For changing $D$ between 0 and $1 \mathrm{in} \sim 12 \mathrm{~h}, k_{\mathrm{p}}$ and $k_{\mathrm{dp}}$ should be of the order of $0,1 \mathrm{~h}^{-1}$.

\section{ATPase reactions}

Both CI and CII domains have ATPase activity, hydrolyzing about 10 ATP molecules in each CI domain and several ATP molecules in each CII domain in a day ${ }^{23,24}$. It is reasonable to consider that ATP is consumed in the CII for supplying a phosphate group in the P process ${ }^{40}$, but the reason for the ATP consumption in the CI has been elusive. With the present treatment $D(k, t)$ implicitly represents the ATPase reactions in the CII, and we more focus on the ATPase reactions in the CI. We consider the case ATP is abundant in the solution; therefore, the probability that the CI binds no nucleotide is small. Hence, we use the variable $q(k, t)=\frac{1}{6} \sum_{i=1}^{6} q(i ; k, t)$ with

$$
q(i ; k, t)= \begin{cases}1 & (i \text { th CI in the } k \text { th KaiC binds ADP }), \\ 0 & (i \text { th CI in the } k \text { th KaiC binds ATP }) .\end{cases}
$$

The ADP release and the subsequent ATP binding are the transition from $q(i ; k, t)=1$ to 0 , and hydrolysis of the bound ATP is the transition from $q(i ; k, t)=0$ to 1 . We simulate the stochastic ADP release and the ATP hydrolysis by treating $q(i ; k, t)$ as a stochastic variable changing with the lifetime of the ADP bound state, $\Delta_{\mathrm{ADP}}$, and the frequency of hydrolysis, $f_{\text {hyd }}$. The ATPase activity measured by the amount of the released ADP from KaiC is large in the $\mathrm{P}$ process ${ }^{23}$, and the $X \approx 0(X \approx 1)$ structure binds ADP (ATP) ${ }^{27}$. These observations are consistent with the assumption that $f_{\text {hyd }}$ is a constant independent of $X(k, t)$ and $\Delta_{\mathrm{ADP}}(k, t)$ is a decreasing function of $X(k, t)$ as

$$
\Delta_{\mathrm{ADP}}(k, t)=\Delta_{\mathrm{ADP}}^{0}\left[1-\tanh \left((2 X(k, t)-1) / C_{X}\right)\right],
$$

where $\Delta_{\mathrm{ADP}}^{0}$ is a constant determining the timescale and $C_{X}$ is a constant determining the seisitivity to the structure.

\section{Feedback coupling through structural change}

Allosteric transitions in protein oligomers typically have a timescale of $10^{-3} \sim 10^{-2} \mathrm{~s}^{63}$, and we assume a similar timescale in the present problem. Because the other reactions are much slower, we describe the KaiC structure as in quasi-equilibrium by treating the chemical states as the quasi-static constraints. Considering that the structural variable $X$ behaves as an Ising variable and the chemical states $R(k, t)$ should work like the external field acting on $X$, we should have the expression, $X(k, t)=\frac{1}{2}\left(1+\tanh \left[R(k, t) /\left(k_{\mathrm{B}} T\right)\right]\right)$. Expanding $R(k, t)$ up to the linear terms of chemical states represented by $P_{\mathrm{C}_{6} \mathrm{~A}_{2}}, P_{\mathrm{C}_{6} \mathrm{~B}_{i} \mathrm{~A}_{2 j}}$, $D(k, t)$, and $q(k, t)$, we have

$$
\begin{aligned}
R(k, t)= & d_{0}+d_{1} P_{\mathrm{C}_{6} \mathrm{~A}_{2}}(k, t)-d_{2} \sum_{i=0}^{6} \sum_{j=0}^{i} P_{\mathrm{C}_{6} \mathrm{~B}_{i} \mathrm{~A}_{2 j}}(k, t) \\
& -d_{3}[D(k, t)-(1-D(k, t))]-d_{4} F(q(k, t)),
\end{aligned}
$$

where $d_{0}$ is a constant to determine the average structure, and $d_{1}, d_{2}, d_{3}$, and $d_{4}$ are constants defining the strength of the feedback coupling. We assume $d_{1}>0$, which stabilizes the $X \approx 1$ structure when KaiA binds on the CII, and $d_{2}>0$, which stabilizes the $X \approx 0$ structure when KaiB binds on the CI. Then, with the definitions of $h_{\mathrm{A}}(k, t), f_{\mathrm{A}}(k, t), h_{\mathrm{B}}(k, t)$, and $f_{\mathrm{B}}(k, t)$, we see that binding reactions constitute the positive feedback loops to stabilize the two states, the $X \approx 1$ and $X \approx 0$ states. 
We use a constant $d_{3}>0$ in Eq. 4 , which destabilizes the $X \approx 1$ state when phosphorylated $(D \approx 1)$ and destabilizes the $X \approx 0$ state when dephosphorylated $(D \approx 0)$, This assumption is consistent with the X-ray crystallography analysis that Ser431 is phosphorylated in the $X \approx 0$ structure while dephosphorylated in the $X \approx 1$ structure $^{27}$. Because $k_{\mathrm{p}}$ and $k_{\mathrm{dp}}$ in Eq. 1 are small, this destabilization is a slow process gradually proceeding after the structural transition. Therefore, the $d_{3}$ term represents the time-delayed negative feedback loops to drive the oscillations between the $X \approx 1$ and $X \approx 0$ states.

$d_{4} F(q(k, t))$ in Eq. 4 represents the coupling of structure with the ATPase reactions, which largely determines the balance between positive and negative feedback effects. This coupling was inferred from the observations that the ATP hydrolysis is necessary for binding $\mathrm{KaiB}$ to $\mathrm{KaiC}^{51,54,64,65}$, and that the structure is modified upon ATP hydrolysis ${ }^{24,27,60,66}$. With $F(q(k, t))=q(k, t) X(k, t)-(1-q(k, t))(1-X(k, t))$ and $d_{4}>0$, the ADP binding on the CI destabilizes the $X \approx 1$ state, while the ATP binding destabilizes the $X \approx 0$ state. Therefore, the stochastic ATP hydrolysis triggers the transition to the $X \approx 0$ state, and the stochastic release of ADP with the subsequent ATP binding triggers the transition to the $X \approx 1$ state (Fig. 1).

The model describes the positive feedback between the structure and the binding/unbinding of KaiA and KaiB to/from KaiC (the $d_{1}$ and $d_{2}$ terms), the negative feedback between the structure and the P/dP process (the $d_{3}$ term), and the transitiontriggering effects of the ATPase reactions (the $d_{4}$ term). These couplings generate the cooperative chemical and structural oscillations in individual KaiC molecules.

\section{Communication among many KaiC molecules at the ensemble level}

We simulated the ensemble of $N=1000$ or 2000 KaiC hexamers. For $N=1000$ and $V=3 \times 10^{-15} l$, the concentration of KaiC is $C_{\mathrm{T}}=3.3 \mu \mathrm{M}$ on a monomer basis, which is near to the value $3.5 \mu \mathrm{M}$ often used in experiments. We assumed the ratio $A_{\mathrm{T}}: B_{\mathrm{T}}: C_{\mathrm{T}}=1: 3: 3$ as used in many experiments ${ }^{23,54,67}$, where $A_{\mathrm{T}}$ and $B_{\mathrm{T}}$ are total concentrations of KaiA and KaiB on a monomer basis. The system was described by variables, $P_{\mathrm{C}_{6} \mathrm{~A}_{2}}(k, t), P_{\mathrm{C}_{6} \mathrm{~B}_{i} \mathrm{~A}_{2 j}}(k, t), D(k, t), X(k, t), q(k, t), x_{A}(t)$ and $x_{B}(t)$, with $k=1, \ldots, N . P_{\mathrm{C}_{6} \mathrm{~B}_{i} \mathrm{~A}_{2 j}}(k, t)$ and $D(k, t)$ were calculated by numerically integrating the kinetic equations, and $P_{\mathrm{C}_{6} \mathrm{~A}_{2}}(k, t)$ and $X(k, t)$ were calculated with the quasi-equilibrium approximation at each time step. $q(k, t)$ was calculated by simulating the stochastic transitions of $q(i ; k, t)$ between 0 and 1 with frequencies $\Delta_{\mathrm{ADP}}^{-1}$ and $f_{\text {hyd }}$. Concentrations of free unbound KaiA dimer and KaiB monomer, $x_{A}(t)$ and $x_{B}(t)$, were calculated at each time step from the following equations of conservation,

$$
\begin{aligned}
x_{A}(t) & +\frac{1}{V} \sum_{k=1}^{N} P_{\mathrm{C}_{6} \mathrm{~A}_{2}}(k, t) \\
& +\frac{1}{V} \sum_{k=1}^{N} \sum_{i=1}^{6} \sum_{j=1}^{i} j P_{\mathrm{C}_{6} \mathrm{~B}_{i} \mathrm{~A}_{2 j}}(k, t)=A_{\mathrm{T}} / 2,
\end{aligned}
$$

and $x_{B}(t)+\frac{1}{V} \sum_{k=1}^{N} \sum_{i=1}^{6} i P_{\mathrm{C}_{6} \mathrm{~B}_{i}}(k, t)=B_{\mathrm{T}}$. Competition between the 2 nd and 3 rd terms of the 1.h.s. of Eq. 5 provides communication among KaiC molecules leading to the synchronization. See the Methods section for details.

\section{Results}

\section{Single-molecule and ensemble-level oscillations}

Figure 2 shows the calculated example oscillations. A KaiC hexamer arbitrarily chosen from the simulated ensemble of $N=1000$ hexamers shows the structural transitions between the states $X(k, t) \approx 1$ and $X(k, t) \approx 0$ (Fig. 2A). The nucleotidebinding state in the CI ring $q(k, t)$ also exhibits transitions between the state rapidly fluctuating around $q(k, t) \approx 0.3$ and the state around $q(k, t) \approx 0.5$. The phosphorylation level $D(k, t)$ follows these switching transitions with the slower rates of the $\mathrm{P} / \mathrm{dP}$ reactions; $D$ increases in the $X \approx 1$ state and decreases in the $X \approx 0$ state, showing saw-tooth oscillations.

At the ensemble level, these fluctuating oscillations in individual molecules are averaged, resulting in the regular oscillations of $\bar{D}(t)=\frac{1}{N} \sum_{k=1}^{N} D(k, t), \bar{X}(t)=\frac{1}{N} \sum_{k=1}^{N} X(k, t)$, and $\bar{q}(t)=\frac{1}{N} \sum_{k=1}^{N} q(k, t)$ (Fig. 2B). The ensemble-averaged rate of the ADP release from KaiC, $\bar{q}_{r}(t)$ (Methods), is large during the $\mathrm{P}$ phase as observed experimentally ${ }^{23}$, and the ADP binding probability $\bar{q}(t)$ is large during the $\mathrm{dP}$ phase consistently with the experimental observations ${ }^{27}$. In this way, the model reproduces the stable circadian oscillations at the ensemble level averaging the synchronized individual KaiC oscillations. 

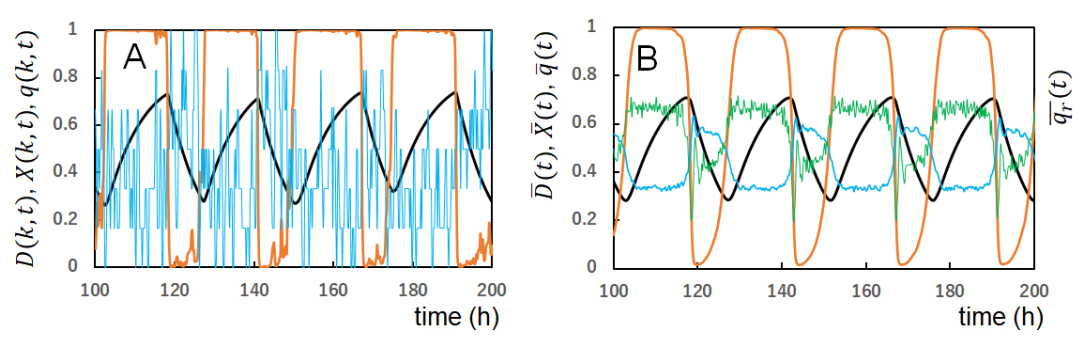

Figure 2. Example oscillations of the simulated KaiABC system. (A) The singlel-molecule oscillations of the phosphorylation level $D(k, t)$ (black), the structural state $X(k, t)$ (orange), and the ADP binding probability on the CI $q(k, t)$ (blue) of an arbitrarily chosen $k$ th KaiC hexamer. (B) The ensemble-averaged oscillations of the phosphorylation level $\bar{D}(t)$ (black), the structural state $\bar{X}(t)$, the binding probability of ADP on the CI $\bar{q}(t)$ (blue), and the ATPase activity measured by the amount of the released ADP $\bar{q}_{r}(t)$ (green). $N=1000$ at temperature $T_{0}=30^{\circ} \mathrm{C}$.

\section{Modifications of the feedback strength}

The binding of KaiA or KaiB may induce the global change of each KaiC subunit, shifting the position and orientation of subunit, whose effects represented by $d_{1}$ and $d_{2}$ in Eq. 4 should be insensitive to the single-residue substitution at the CI-CII interface. On the other hand, $\mathrm{P} / \mathrm{dP}$ in the $\mathrm{CII}$ or the ATPase reaction in the $\mathrm{CI}$ is the local atomic change around the phosphate group, whose effects are transmitted through chains of electrostatic and volume-excluding interactions, producing the allosteric communication between the $\mathrm{CI}$ and $\mathrm{CII}^{27,66}$. This process should be sensitive to the atomic interactions at the CI-CII interface and hence sensitive to the single-residue substitution. Here, we assume that substituting a CI-CII interface residue to the smaller volume one is represented by the decrease in $d_{3}$ and $d_{4}$ in Eq. 4 with a scaling factor $s<1$ to $s d_{3}$ and $s d_{4}$ while $d_{1}$ and $d_{2}$ in Eq. 4 being kept in their original values. With $s<1$, the simulated phosphorylation level oscillations indeed show the large amplitude and long period as expected from the decrease in the negative feedback strength, while the amplitude is small and the period is short for $s>1$ (Fig. 3A). On the contrary, with positive feedback enhancement with a scaling factor $s>1$, changing $d_{1}$ and $d_{2}$ to $s d_{1}$ and $s d_{2}$ enlarges the amplitude and period, while they are reduced when $s<1$ (Fig. 3B).
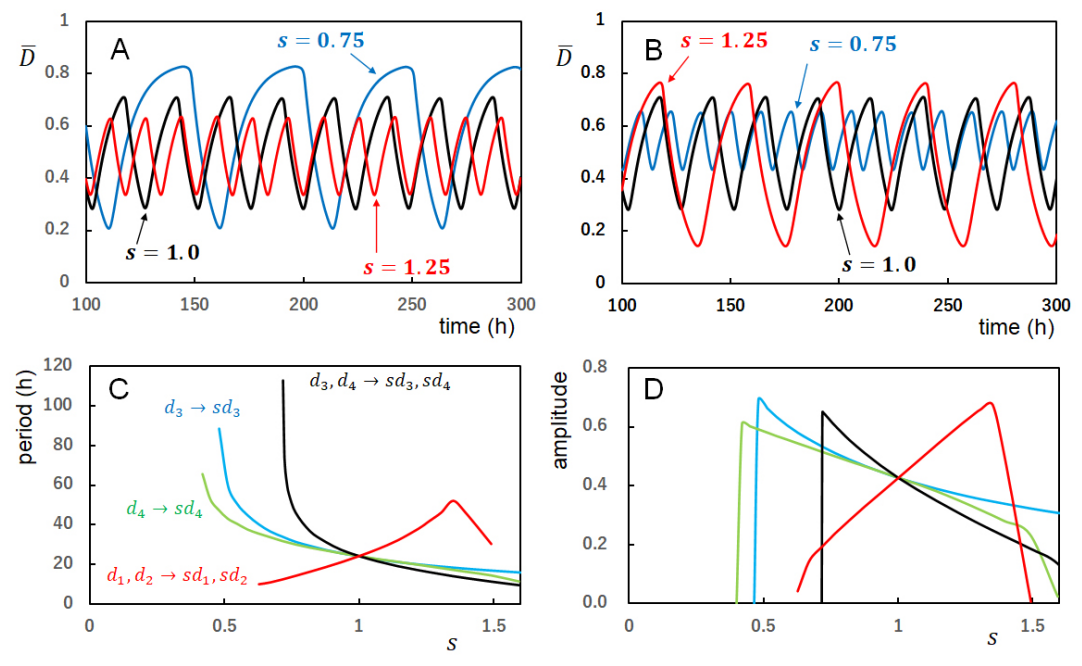

Figure 3. Effects of modification of the reaction-structure feedback strength. (A) and (B) Example oscillations of the ensemble-averaged phosphorylation level $\bar{D}(t)$ with a varying factor $s$. (A) The negative feedback coupling between structure and reactions was modified; the coupling with the P/dP reactions $d_{3}$ and the one with the ATPase reactions $d_{4}$ were modified to $s d_{3}$ and $s d_{4}$ with $s=0.75$ (blue), 1.0 (black), and 1.25 (red). (B) The positive feedback coupling between structure and the binding/unbinding reactions was modified; the coupling with the KaiA binding/unbinding $d_{1}$ and the one with the KaiB binding/unbinding $d_{2}$ were modified to $s d_{1}$ and $s d_{2}$ with $s=0.75$ (blue), 1.0 (black), and 1.25 (red). Period (C) and amplitude (D) of the simulated $\bar{D}(t)$ oscillations plotted as functions of the scaling factor $s$ for modifying $d_{3}$ to $s d_{3}$ while $d_{1}, d_{2}$, and $d_{4}$ being kept constant (blue), $d_{4}$ to $s d_{4}$ while $d_{1}, d_{2}$, and $d_{3}$ being kept constant (green), $d_{3}$ and $d_{4}$ to $s d_{3}$ and $s d_{4}$ while $d_{1}$ and $d_{2}$ being kept constant (black), and $d_{1}$ and $d_{2}$ to $s d_{1}$ and $s d_{2}$ while $d_{3}$ and $d_{4}$ being kept constant (red). $N=1000$ in $\mathbf{A}$ and $\mathbf{B}$, and $N=2000$ in $\mathbf{C}$ and $\mathbf{D}$. Temperature was $T_{0}=30^{\circ} \mathrm{C}$. 
Table 1. Temperature dependence/independence of specific parameters.

\begin{tabular}{|c|c|c|}
\hline Rules & $T$-dependence* & Physical implications \\
\hline Rule 1 & $\begin{array}{l}d_{3}(T)=\frac{d_{3}\left(T_{0}\right)}{s\left(\Delta E_{\mathrm{f}} ; T, T_{0}\right)} \\
d_{4}(T)=\frac{d_{4}\left(T_{0}\right)}{s\left(\Delta E_{\mathrm{f}} ; T, T_{0}\right)}\end{array}$ & $\begin{array}{l}\text { Thermally attenuated } \\
\text { structure-P/dP feedback } \\
\text { Thermally attenuated } \\
\text { structure-ATPase coupling }\end{array}$ \\
\hline Rule 2 & $\begin{array}{l}h_{\mathrm{B}}(T) \propto s\left(\Delta E_{0} ; T, T_{0}\right) \\
\times s\left(\Delta E_{\mathrm{fs}-\mathrm{gs}} ; T, T_{0}\right)\end{array}$ & $\begin{array}{l}\text { Thermally activated } \\
\text { KaiB fold }\end{array}$ \\
\hline Rule 3 & $\begin{array}{l}\Delta_{\mathrm{ADP}}^{0}(T)=\Delta_{\mathrm{ADP}}^{0}\left(T_{0}\right) \\
f_{\text {hyd }}(T)=f_{\text {hyd }}\left(T_{0}\right)\end{array}$ & $\begin{array}{l}T \text {-insensitive lifetime } \\
\text { of the ADP bound state } \\
T \text {-insensitive frequency } \\
\text { of ATP hydrolysis }\end{array}$ \\
\hline $\begin{array}{l}\text { Case A } \\
\text { Case B }\end{array}$ & $\begin{array}{l}\text { Rule } 1+\text { Rule } 2+\text { Rule } 3 \\
\text { Rule } 1+\text { Rule } 3\end{array}$ & $\Delta E_{\mathrm{fs}-\mathrm{gs}}=0$ \\
\hline Case C & Rule $1+$ Rule 2 & $\begin{array}{l}\Delta_{\mathrm{ADP}}^{0}(T)^{-1} \propto s\left(\Delta E_{0} ; T, T_{0}\right) \\
f_{\mathrm{hyd}}(T) \propto s\left(\Delta E_{0} ; T, T_{0}\right)\end{array}$ \\
\hline Case D & Rule 2+Rule 3 & $\Delta E_{\mathrm{f}}=0$ \\
\hline
\end{tabular}

These effects of the feedback strength modifications were systematically examined in Figs. 3C and 3D. When $d_{3}$ is scaled to $s d_{3}$ with $d_{4}, d_{1}$, and $d_{2}$ kept constant, the amplitude and period are extended as $s$ is decreased. When $s \lesssim 0.5$, the oscillations disappear as the system is caught at the $X \approx 1$ state losing the negative feedback destabilization of the $X \approx 1$ state. Similar behaviors were found when the structure-ATPase coupling was changed to $s d_{4}$ with $d_{3}, d_{1}$, and $d_{2}$ kept constant. Thus, the ATPase reactions give similar effects to the negative feedback in the present model. With the combined change to $s d_{3}$ and $s d_{4}$, the period change is more prominent, ranging from $9 \mathrm{~h}$ to $118 \mathrm{~h}$ (Fig. 3C), which explains the observed ten-times period change induced by the single-residue substitution at the CI-CII interface ${ }^{26}$. Modifying the positive feedback strength to $s d_{1}$ and $s d_{2}$ shows that the oscillations disappear when the positive feedback is too weak or too strong (Fig. 3D). The period and amplitude are enlarged as $s$ increases in between these boundaries of positive feedback strength (Fig. 3C).

\section{Thermal loosening of the strcutural coupling explains temperature compensation}

We propose that the increased structural fluctuations of KaiC in the higher temperature should weaken the interactions at the CI-CII interface to decrease $d_{3}$ and $d_{4}$ in Eq. 4, producing similar effects to the single-residue substitution (Fig. 3A). These effects should enlarge the amplitude and period, compensating for the accelerated reactions in high temperatures. Here, we examine our hypothesis by simulating the oscillations in various temperatures.

Assuming that the fluctuations are proportinal to $\exp \left(-\Delta E_{\mathrm{f}} /\left(k_{\mathrm{B}} T\right)\right)$ with a constant $\Delta E_{\mathrm{f}}$, the feedback coupling strength at temperature $T, d_{3}(T)$ and $d_{4}(T)$, should be modified from those in the standard temperature $T_{0}=30^{\circ} \mathrm{C}$ as $d_{3}(T)=$ $d_{3}\left(T_{0}\right) / s\left(E_{\mathrm{f}} ; T, T_{0}\right)$ and $d_{4}(T)=d_{4}\left(T_{0}\right) / s\left(E_{\mathrm{f}} ; T, T_{0}\right)$;

$$
s\left(\Delta E_{\mathrm{f}} ; T, T_{0}\right)=\exp \left(-\Delta E_{\mathrm{f}} /\left(k_{\mathrm{B}} T\right)+\Delta E_{\mathrm{f}} /\left(k_{\mathrm{B}} T_{0}\right)\right) .
$$

We call this temperature dependence of $d_{3}(T)$ and $d_{4}(T)$ Rule 1. Another rule comes from the activation energy $\Delta E_{\mathrm{fs}-\mathrm{gs}}$ for the KaiB fold transformation. Assumption of $\Delta E_{\mathrm{fs}-\mathrm{gs}}>0$ should affect the binding kinetics of KaiB, and we call this assumption Rule 2. The other assumption corresponds to the temperature insensitivity of the ATPase reactions as observed in experiments ${ }^{23,68}$. We assume the temperature-insensitive ATPase reactions by imposing $\Delta_{\mathrm{ADP}}^{0}(T)=\Delta_{\mathrm{ADP}}^{0}\left(T_{0}\right)$ and $f_{\text {hyd }}(T)=$ $f_{\text {hyd }}\left(T_{0}\right)$, where $\Delta_{\mathrm{ADP}}^{0}(T)$ is a constant to determine the lifetime of the ADP bound state (Eq. 3 ), and $f_{\text {hyd }}(T)$ is the hydrolysis frequency of the bound ATP. We call this assumption Rule 3. These three rules are summarized in Table 1.

The model has seven rate constants (Table 2) other than the three rate constants, $h_{\mathrm{B}}, \Delta_{\mathrm{ADP}}^{0}{ }^{-1}$, and $f_{\text {hyd }}$, discussed in Table 1. Each of seven rate constants should depend on temperature in each distinctive way. However, for examining the hypothesis transparently, we assume a straightforward case of the same activation energy $E_{0}$ in seven rate constants, which leads to the same temperature dependence as $k_{\mathrm{dp}}(T)=s\left(\Delta E_{0} ; T, T_{0}\right) k_{\mathrm{dp}}\left(T_{0}\right)$, etc., where $s$ is defined in Eq. 6 . In the case we do not impose any of Rule 1 , Rule 2 , or Rule 3 by assuming $\Delta_{\mathrm{ADP}}^{0}{ }^{-1}(T)=s\left(\Delta E_{0} ; T, T_{0}\right) \Delta_{\mathrm{ADP}}^{0}{ }^{-1}\left(T_{0}\right)$ and $f_{\text {hyd }}(T)=s\left(\Delta E_{0} ; T, T_{0}\right) f_{\text {hyd }}\left(T_{0}\right)$, all 

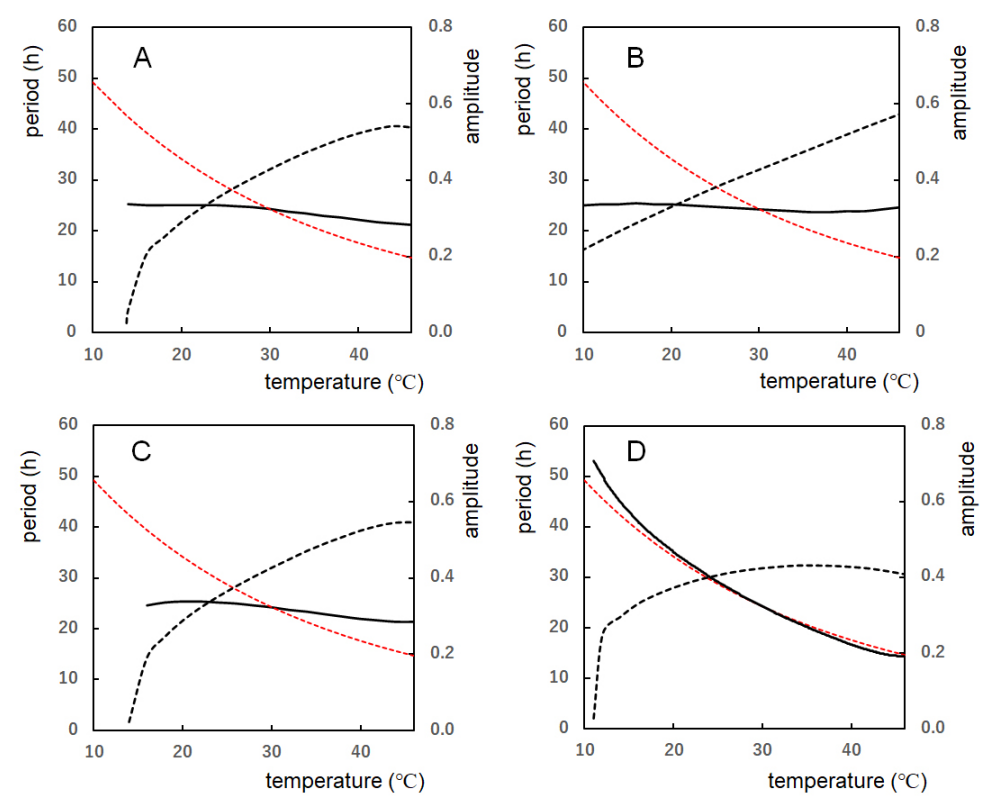

Figure 4. Temperature dependence of the period and amplitude of the simulated oscillations. Period (solid line) and amplitude (dashed line) of the ensemble-averaged phosphorylation level $\bar{D}(t)$ were calculated as functions of temperature with various modeling rules. Compared is the relative change of $1 /$ rate of the process having the $10 k_{\mathrm{B}} T_{0}$ activation energy (red dotted line). (A) Rule 1 (thermal attenuation of the negative feedback coupling), Rule 2 (thermal transformation of KaiB to a bindable conformation), and Rule 3 (temperature-insensitive ATPase reactions) applied (Case A). (B) Rule 1 and Rule 3 applied (Case B). (C) Rule 1 and Rule 2 applied (Case C). (D) Rule 2 and Rule 3 applied (Case D). $N=2000$.

10 rate constants are scaled in the same way, which is almost the same as the scaling in time units, making the oscillation period approximately proportional to $s\left(E_{0} ; T, T_{0}\right)^{-1}$. This homogeneous activation without thermal attenuation of feedback should result in $Q_{10}=\left(\right.$ period in $\left.T_{0}-5^{\circ} \mathrm{C}\right) /\left(\right.$ period in $\left.T_{0}+5^{\circ} \mathrm{C}\right) \approx s\left(E_{0} ; T_{0}+5, T_{0}-5\right) \approx 1.4$ for $E_{0}=10 k_{\mathrm{B}} T_{0}$ and $Q_{10} \approx 2$ for $E_{0}=20 k_{\mathrm{B}} T_{0}$. The purpose of the present subsection is to show that $\left|Q_{10}-1\right| \lesssim 0.1$, or the oscillations are temperature compensated when we assume the three rules in Table 1 even in the case $E_{0}=10 k_{\mathrm{B}} T_{0}$ in other seven rate constants. We also show that Rule 1 plays a dominant role, and temperature compensation is realized without imposing Rule 2 or 3; temperature compensation is realized if Rule 1 is satisfied even when $E_{0}=10 k_{\mathrm{B}} T_{0}$ is used for all ten rate constants.

In order to distinguish the roles of three rules, we examined four cases (Table 1). All three Rules apply in Case A (Fig. 4A), Rules 1 and 3 in Case B (Fig. 4B), Rules 1 and 2 in Case C (Fig. 4C), and Rules 2 and 3 in Case D (Fig. 4D). The period is temperature compensated in Case A $\left(Q_{10}=1.08\right)$, Case B $\left(Q_{10}=1.04\right)$, and Case $\mathrm{C}\left(Q_{10}=1.08\right)$, while the period shows a distinct temperature dependence in Case $\mathrm{D}\left(Q_{10}=1.39\right)$, showing that Rule 1 plays a dominant role in temperature compensation. In Cases A, C, and D, amplitude sharply decreases as temperature decreases below $20^{\circ} \mathrm{C}$ as observed experimentally ${ }^{69}$, showing Rule 2 is essential for explaining the temperature dependence of the amplitude; oscillations disappear with insufficient KaiB binding in low temperature. We should note that Rule 3, temperature insensitivity of ATPase reactions, does not play a significant role in the present examination (Fig. 4C); we discuss more on this point in the next subsection.

A dominant role of Rule 1 is evident also from calculations with the varied temperature dependence of the structural fluctuations. $Q_{10}$ decreases as $\Delta E_{\mathrm{f}}$ increases (Fig. 5A); $Q_{10}=1.39\left(\Delta E_{\mathrm{f}}=0\right), 1.21\left(\Delta E_{\mathrm{f}}=3 k_{\mathrm{B}} T_{0}\right), 1.01\left(\Delta E_{\mathrm{f}}=6 k_{\mathrm{B}} T_{0}\right)$, and $0.84\left(\Delta E_{\mathrm{f}}=9 k_{\mathrm{B}} T_{0}\right)$; showing the temperature compensation with $\Delta E_{\mathrm{f}}=6 k_{\mathrm{B}} T_{0}$, and the distinct overcompensation with $9 k_{\mathrm{B}} T_{0}$. A drop of the amplitude in the low temperature regime takes place at the higher temperature as $\Delta E_{\mathrm{f}}$ increases (Fig. 5B), consistently with the expected period-amplitude correlation.

\section{Effects of the ATPase reactions}

In order to clarify the effects of the ATPase reactions on the oscillations, we scaled the rate constants of the ATPase reactions in the present model. Fig. 6A shows the amplitude and period calculated by modifying the inverse lifetime of the ADP bound state, $\Delta_{\mathrm{ADP}}^{0}{ }^{-1}$, and the hydrolysis frequency of the bound ATP, $f_{\text {hyd }}$. They were scaled by a factor $s_{\mathrm{a}}$ in two different ways; in case I, they were scaled as $s_{\mathrm{a}}\left(\Delta_{\mathrm{ADP}}^{0}\right)^{-1}$ and $s_{\mathrm{a}} f_{\text {hyd }}$, and in case II, $\left(s_{\mathrm{a}} \Delta_{\mathrm{ADP}}^{0}\right)^{-1}$ and $s_{\mathrm{a}} f_{\text {hyd }}$. In case I, period and amplitude only slightly 

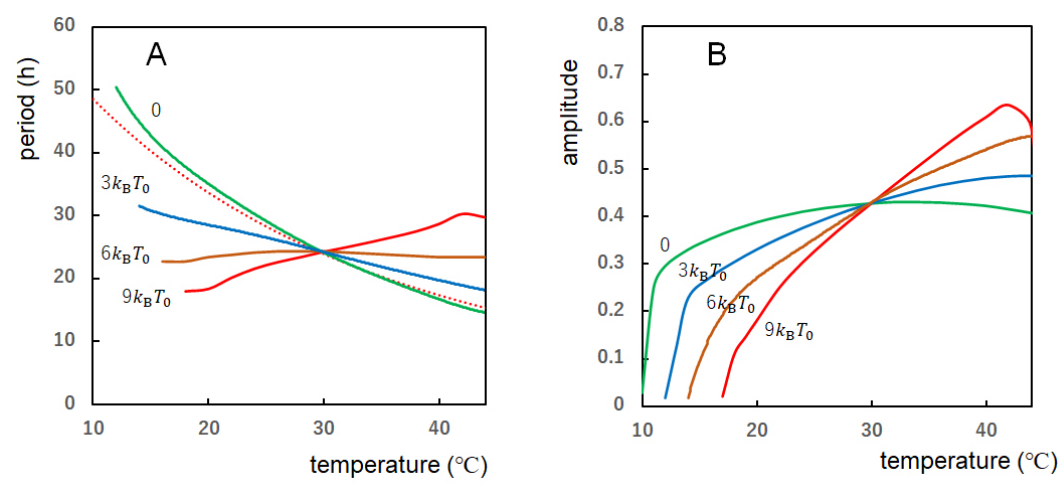

Figure 5. Temperature dependence of the period and amplitude simulated with a different activation energy of structural fluctuations. Period and amplitude of the ensemble-averaged phosphorylation level $\bar{D}(t)$ were calculated with various values of the activation energy of structural fluctuations $\Delta E_{\mathrm{f}}$ with $\Delta E_{\mathrm{f}}=0$ (green), $3 k_{\mathrm{B}} T_{0}$ (blue), $6 k_{\mathrm{B}} T_{0}$ (brown), and $9 k_{\mathrm{B}} T_{0}$ (red) with $T_{0}=30^{\circ} \mathrm{C}$. (A) Period plotted for each $\Delta E_{\mathrm{f}}$ as a function of temperature. Compared is the relative change of $1 /$ rate of the process having the $10 k_{\mathrm{B}} T_{0}$ activation energy (red dotted line). (B) Amplitude plotted for each $\Delta E_{\mathrm{f}}$ as a function of temperature. $N=2000$.
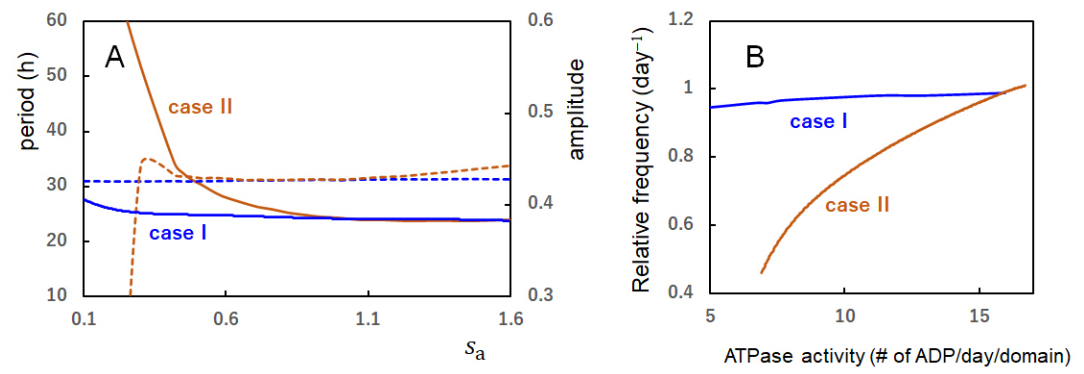

Figure 6. Oscillations and the ATPase activity calculated with the modified rate constants of the ATPase reactions. Two ways of the modification, case I and case II, were tested on the inverse lifetime of the ADP bound state, $\Delta_{\mathrm{ADP}}^{0}{ }^{-1}$, and the frequency of hydrolysis of the bound ATP, $f_{\text {hyd }}$. In case I (blue), the rate constants were scaled as $s_{\mathrm{a}}\left(\Delta_{\mathrm{ADP}}^{0}\right)^{-1}$ and $s_{\mathrm{a}} f_{\text {hyd }}$, and in case II (brown), $\left(s_{\mathrm{a}} \Delta_{\mathrm{ADP}}^{0}\right)^{-1}$ and $s_{\mathrm{a}} f_{\text {hyd }}$ (A) The period (solid line) and amplitude (dashed line) of the ensemble-averaged phosphorylation level $\bar{D}(t)$ were calculated as functions of $s_{\mathrm{a}}$. (B) The frequency (inverse period) of oscillations of $\bar{D}(t)$ is compared with the ATPase activity (in units of the number of released ADP molecules from a CI domain in a day). The ATPase activity was calculated in the nonoscillatory condition in the absence of KaiA and KaiB. Temperature is $T_{0}$ and $N=2000$.

depend on $s_{\mathrm{a}}$. This insensitivity to the scaling corresponds to the temperature insensitivity shown in Fig. 4C, where we did not use Rule 3 but scaled the constants as $s\left(\Delta E_{0} ; T, T_{0}\right)\left(\Delta_{\mathrm{ADP}}^{0}\right)^{-1}$ and $s\left(\Delta E_{0} ; T, T_{0}\right) f_{\text {hyd }}$. The insensitivity in case I and Fig. 4C indicates that the change in the ATPase reaction rates does not affect the period when they were changed with the constraint $\Delta_{\mathrm{ADP}} \cdot f_{\text {hyd }}=$ const. The ATPase reactions should affect the period in two ways: The probability that the CI binds ADP during the $\mathrm{P}$ phase is correlated to how the $X \approx 1$ state is destabilized, and the probability that the CI binds ATP during the $\mathrm{dP}$ phase is correlated to how the $X \approx 0$ state is destabilized. However, changing the rates under the constraint $\Delta_{\mathrm{ADP}} \cdot f_{\text {hyd }}=$ const. does not affect these probabilities. Therefore, the period was insensitive to the changes in the rate constants under the constraint $\Delta_{\mathrm{ADP}} \cdot f_{\text {hyd }}=$ const.

In case II, this constraint is not satisfied, and the change in the rate constants brings about a significant change in the period (Fig. 6A). We examined the correlation between the oscillation frequency (i.e., 1/period) and the ATPase activity calculated in the nonoscillatory condition in the absence of KaiA and KaiB. Fig. 6B shows a clear correlation between the oscillation frequency and the ATPase activity in case II as experimentally observed ${ }^{23,24}$. The period is insensitive to the change in the ATPase rates only when the specific constraint is satisfied. This result suggests that the strategy to keep the specific constraint has been avoided in evolutionary design. Instead, the more general strategy of the temperature-insensitive ATPase reactions might have been evolutionarily realized in $\mathrm{KaiC}^{23,68}$ as in the TTO regulator, $\mathrm{CKI} \varepsilon / \delta$, in mammals ${ }^{22}$. 

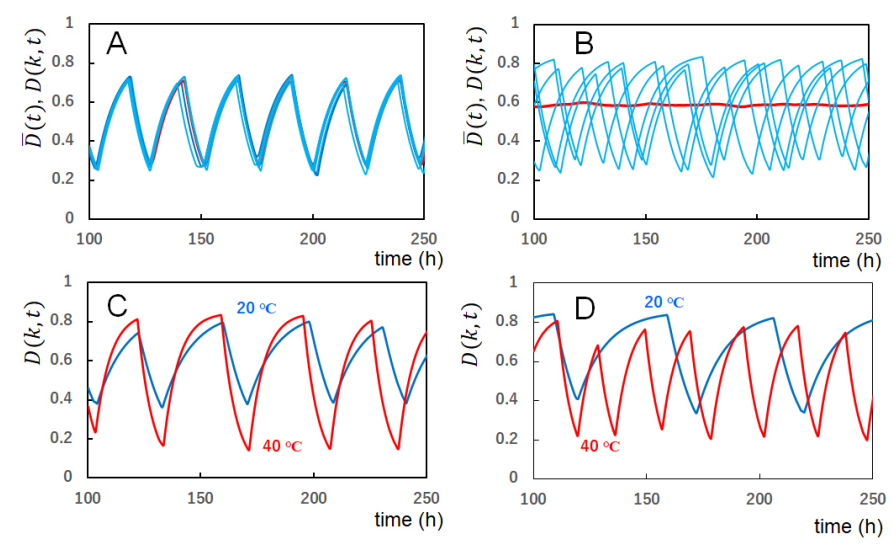

Figure 7. Temperature compensation of oscillations of individual molecules. In $\mathbf{A}$ and $\mathbf{B}$, oscillations of the ensemble-averaged phosphorylation level $\bar{D}(t)$ (red) are compared with five individual oscillations $D(k, t)$ of molecules arbitrarily chosen from the ensemble (blue). $T=T_{0}$. (A) $h_{\mathrm{AB}}$ was kept in the standard value (Table 2). (B) $h_{\mathrm{AB}}$ was reduced to $1 / 200$ of the standard value. (C) Oscillations of $D(k, t)$ of an arbitrarily chosen single molecule at $T=40^{\circ} \mathrm{C}$ (red) and $T=20^{\circ} \mathrm{C}$ (blue). Three rules, Rule 1 (thermal attenuation of the reaction-structure feedback coupling with $\Delta E_{\mathrm{f}}=5 k_{\mathrm{B}} T$ ), Rule 2 (thermal activation of KaiB transformation), and Rule 3 (temperature insensitivity of the ATPase reactions), were assumed. (D) Oscillations of $D(k, t)$ of an arbitrarily chosen single molecule at $T=40^{\circ} \mathrm{C}$ (red) and $T=20^{\circ} \mathrm{C}$ (blue). Rule 2 and Rule 3 were assumed, while Rule 1 was not adopted with $\Delta E_{\mathrm{f}}=0$. In $\mathbf{C}$ and $\mathbf{D}, h_{\mathrm{AB}}$ was reduced to 1/200 of the standard value. $N=1000$.

\section{Discussion}

Using the model describing the feedback coupling among reactions and structural transitions in individual KaiC molecules and synchronization of many KaiC molecules, we showed that weakening the negative feedback strength extends the amplitude and lengthens the period of oscillations in the KaiABC system. This weakening can explain the observed wide range of period modification induced by the single-residue substitution at the CI-CII interface of $\mathrm{KaiC}^{26}$. We hypothesized that thermal fluctuations induce similar effects to the substitution at the CI-CII interface, which explained the stable temperature compensation in the KaiABC system. The ATPase reactions also affect the period, but the period is insensitive when the ATPase rates are changed under the specific constraint.

A possible test of the thermal weakening of the CI-CII structural coupling is to measure the temperature dependence of the thermal fluctuations of the interface residue with NMR or other spectroscopic methods. The model predicts that the temperature dependence of fluctuations should be anti-correlated with the $Q_{10}$ of the period, and such an anti-correlation was suggested from the recent neutron scattering data ${ }^{68}$.

Another possible test is to observe the single-molecular behavior. In the typical oscillatory condition, oscillations of individual KaiC molecules are synchronized, inducing coherent circadian oscillations at the ensemble level (Fig. 7A). If the synchronization is realized through the sequestration of KaiA into the KaiC-KaiB-KaiA complexes as assumed in the present study, synchronization should be lost when the binding affinity between KaiA and KaiB is reduced. It would be possible to design a KaiB mutant with a low affinity to KaiA, and in the present model, such a mutant is represented by reducing the $h_{\mathrm{AB}}$ value. With this reduction, synchronization is lost, and the ensemble oscillations disappear despite the oscillations with the large amplitude remaining in individual KaiC molecules (Fig. 7B). In such a desynchronized condition, period of individual oscillations is temperature compensated with the temperature-dependent oscillation amplitude in the present model (Fig. 7C). In contrast, when the negative feedback strength does not depend on temperature, individual oscillations are not temperature compensated without showing a significant temperature dependence of amplitude (Fig. 7D). The temperature compensation hypothesis in the present study contrasts with the hypothesis of the competitive KaiA binding reactions ${ }^{25}$. With the latter hypothesis, temperature compensation is induced only from the ensemble level mechanism, not from the individual molecular mechanism, which should be distinguishable with the single-molecule observation in the condition that the synchronization is lost.

Our hypothesis on temperature compensation was based on the observations of the coupling between reactions and structural transitions in the oscillating molecules ${ }^{26,27}$. Investigations of the KaiABC system should highlight the significance of the regulation through reaction-structure coupling in individual molecules. These analyses should help provide innovative methods of atomic design for regulating the system dynamics of an ensemble of molecules. 


\section{Methods}

\section{Multifold feedback coupling in KaiC}

We describe individual KaiC hexamers with coarse-grained variables; the binding state of KaiA on the CII domain, $\theta_{\mathrm{C}_{6}} \mathrm{~A}_{2}(k)$, the binding state of $\mathrm{KaiB}$ and KaiA on the CI domain, $\theta_{\mathrm{C}_{6} \mathrm{~B}_{i} \mathrm{~A}_{2 j}}(k)$, the phosphorylation level, $0 \leq \mathscr{D}(k) \leq 1$, the structural state, $W(k)$, and the nucleotide-binding state, $q(i ; k, t)$. Here,

$$
\begin{aligned}
& \theta_{\mathrm{C}_{6} \mathrm{~A}_{2}}(k)= \begin{cases}1 & (\text { the } \mathrm{CII} \text { of the } k \text { th KaiC binds KaiA) } \\
0 & \text { (otherwise) }\end{cases} \\
& \theta_{\mathrm{C}_{6} \mathrm{~B}_{i} \mathrm{~A}_{2 j}}(k)= \begin{cases}1 & (\# \text { of KaiB and KaiA on the CI of the } k \text { th KaiC are } i \text { and } j), \\
0 & \text { (otherwise), }\end{cases} \\
& W(k)= \begin{cases}1 & \text { (strucutre in the } \mathrm{P} \text { process) } \\
-1 & \text { (structure in the } \mathrm{dP} \text { process) },\end{cases} \\
& \mathscr{D}(k)=\text { (\# of phosphorylated sites in the } k \text { th KaiC) } / 12 \text {, } \\
& q(i ; k)= \begin{cases}1 & (\text { the } i \text { th CI domain of the } k \text { th KaiC haxamer binds ADP) } \\
0 & \text { (the } i \text { th CI domain of the } k \text { th KaiC haxamer binds ATP }) .\end{cases}
\end{aligned}
$$

We consider the system to consist of $N$ KaiC hexamers. Then, the system state at time $t$ is described by a set of vectors, $\vec{\theta}_{\mathrm{C}_{6} \mathrm{~A}_{2}}=\left\{\theta_{\mathrm{C}_{6} \mathrm{~A}_{2}}(1), \theta_{\mathrm{C}_{6} \mathrm{~A}_{2}}(2), \cdots, \theta_{\mathrm{C}_{6} \mathrm{~A}_{2}}(N)\right\}$, etc. The stochastic evolution of the system state is described by the probability distribution,

$$
P_{\text {system }}(t)=P\left(\vec{\theta}_{\mathrm{C}_{6} \mathrm{~A}_{2}}, \vec{\theta}_{\mathrm{C}_{6} \mathrm{~B}_{i} \mathrm{~A}_{2 j}}, \overrightarrow{\mathscr{D}}, \vec{W}, \vec{q}, x_{\mathrm{A}}(t), x_{\mathrm{B}}(t), t\right)
$$

where $x_{\mathrm{A}}(t)$ and $x_{\mathrm{B}}(t)$ are concentrations of free KaiA dimer and KaiB monomer unbound from KaiC, respectively. The structural change takes place in milliseconds or so in usual protein oligomers, and we assume a similar timescale in the present problem. This timescale is much shorter than the timescales of other reactions; therefore, we treat the variable $\vec{W}$ as in quasi-equilibrium. By treating $W(k)$ like the Ising spin under the external field $R(k, t)$, the average of $W(k)$ in quasi-equilibrium is $\bar{W}(k)(t)=\tanh (-\beta R(k, t))$. By intoducing the order parameter of structure, $0 \leq X(k, t) \leq 1$, as $X(k, t)=(\bar{W}(k)(t)+1) / 2$, we have

$$
X(k, t)=\frac{1}{2}(1+\tanh (\beta R(k, t))
$$

showing the same form as in Eq. 4 in the main text. Here, $\beta=1 /\left(k_{\mathrm{B}} T\right)$ is inverse temperature, and $R(k, t)$ is a quasi-equilibrium constraint reflecting the chemical state of the KaiC hexamer at time $t$. The explicit form of $R(k, t)$ represents how the feedback relations among reactions and structure work in the system and is defined after the other variables in Eq. 12 are transformed to an expression suitable to be handled.

We use the mean-field approximation; Eq. 12 is approximated by factorizing $P$ into each degree of freedom as

$$
\begin{aligned}
& P_{\text {system }}(t)= \prod_{k=1}^{N} P\left(\theta_{\mathrm{C}_{6} \mathrm{~A}_{2}}(k), X(k, t), \mathbf{q}(k, t), x_{\mathrm{A}}(t), x_{\mathrm{B}}(t), t\right) \\
& \times \prod_{k=1}^{N} \prod_{i=0}^{6} \prod_{j=1}^{i} P\left(\theta_{\mathrm{C}_{6} \mathrm{~B}_{i} \mathrm{~A}_{2 j}}(k), X(k, t), \mathbf{q}(k, t), x_{\mathrm{A}}(t), x_{\mathrm{B}}(t), t\right) \\
& \times \prod_{k=1}^{N} P\left(\mathscr{D}(k), X(k, t), \mathbf{q}(k, t), x_{\mathrm{A}}(t), x_{\mathrm{B}}(t), t\right),
\end{aligned}
$$

where $\mathbf{q}(k, t)=\{q(1 ; k, t), q(2 ; k, t), \ldots, q(6 ; k, t)\}$ is a six-dimensional vector representing the nucleotide-binding state of each of six CI domains in the $k$ th hexamer. We represent the non-equilibrium consumption of ATP by treating $q(i ; k, t)$ as a stochastic variable taking the value either 1 or 0 depending on whether the CI domain binds $\operatorname{ADP}$ or ATP $(\mathrm{Eq} .11) . X(k, t), x_{\mathrm{A}}(t)$, and $x_{\mathrm{B}}(t)$ are calculated by solving the self-consistent relations explained in the Subsection "Self-consistent relations" in this SI Text. Thus, by dropping the variables, $\mathbf{q}(k, t), X(k, t), x_{\mathrm{A}}(t)$, and $x_{\mathrm{B}}(t)$ from the expression, Eq. 14 is

$$
P_{\text {system }}(t)=\prod_{k=1}^{N}\left[P\left(\theta_{\mathrm{C}_{6} \mathrm{~A}_{2}}(k), t\right)\left(\prod_{i=0}^{6} \prod_{j=1}^{i} P\left(\theta_{\mathrm{C}_{6} \mathrm{~B}_{i} \mathrm{~A}_{2 j}}(k), t\right)\right) P(\mathscr{D}(k), t)\right] .
$$


$P_{\text {system }}(t)$ should obey the master equation representing reactions in the Kai system. In the mean-field approximation, the master equation is reduced to simpler equations similar to the chemical kinetics equations ${ }^{70,71}$. Thus, we consider the equation of KaiA binding/unbinding kinetics for $P\left(\theta_{\mathrm{C}_{6} \mathrm{~A}_{2}}(k), t\right)$, the equations of KaiB and KaiA binding/unbinding kinetics for $P\left(\theta_{\mathrm{C}_{6} \mathrm{~B}_{i} \mathrm{~A}_{2 j}}(k), t\right)$, and the equation of $\mathrm{P} / \mathrm{dP}$ kinetics for $P(\mathscr{D}(k), t)$.

\section{KaiA and KaiB binding/unbinding kinetics}

Writing $P_{\mathrm{C}_{6} \mathrm{~A}_{2}}(k, t)=P\left(\theta_{\mathrm{C}_{6} \mathrm{~A}_{2}}(k)=1, t\right)$, we have the kinetic equation for the KaiA binding and unbinding,

$$
\frac{d}{d t} P_{\mathrm{C}_{6} \mathrm{~A}_{2}}(k, t)=h_{\mathrm{A}} x P_{\mathrm{C}_{6} \mathrm{~B}_{0} \mathrm{~A}_{0}}(k, t)-f_{\mathrm{A}} P_{\mathrm{C}_{6} \mathrm{~A}_{2}}(k, t) .
$$

Because the KaiA binding and unbinding reactions are faster than the other reactions in the present system, we can approximate Eq. 16 with the quasi-equilibrium approximation as $\frac{d}{d t} P_{\mathrm{C}_{6} \mathrm{~A}_{2}}(k, t)=0$. Then, we have

$$
P_{\mathrm{C}_{6} \mathrm{~A}_{2}}(k, t)=x_{\mathrm{A}}(t) g_{\mathrm{C}: \mathrm{A}}(k, t) P_{\mathrm{C}_{6} \mathrm{~B}_{0} \mathrm{~A}_{0}}(k, t),
$$

with $g_{\mathrm{C}: \mathrm{A}}(k, t)=h_{\mathrm{A}}(k, t) / f_{\mathrm{A}}(k, t)$. As discussed in the main text, $h_{\mathrm{A}}$ should be an increasing function of $X(k, t)$ and $f_{\mathrm{A}}$ is a decreasing function of $X(k, t)$. We use the form,

$$
\begin{aligned}
& h_{\mathrm{A}}(k, t)=h_{\mathrm{A} 0}\left[1+\tanh \left(\frac{2 X(k, t)-1}{A_{X}}\right)\right], \\
& f_{\mathrm{A}}(k, t)=f_{\mathrm{A} 0}\left[1-\tanh \left(\frac{2 X(k, t)-1}{A_{X}}\right)\right],
\end{aligned}
$$

where $h_{\mathrm{A} 0}$ and $f_{\mathrm{A} 0}$ are the rate cosnstants to determine the time scale and $A_{X}>0$ is a constant to determine the sensitvity to the structure.

In a similar way, by writing $P_{\mathrm{C}_{6} \mathrm{~B}_{i} \mathrm{~A}_{2 j}}(k, t)=P\left(\theta_{\mathrm{C}_{6} \mathrm{~B}_{i} \mathrm{~A}_{2 j}}(k)=1, t\right)$, the binding and unbinding of KaiA to and from KaiB should be in quasi-equilibrium, leading to

$$
P_{\mathrm{C}_{6} \mathrm{~B}_{i} \mathrm{~A}_{2 j}}(k, t)=x_{\mathrm{A}}(t) g_{\mathrm{CB}: \mathrm{A}} P_{\mathrm{C}_{6} \mathrm{~B}_{i} \mathrm{~A}_{2(j-1)}}(k, t),
$$

with $g_{\mathrm{CB}: \mathrm{A}}=h_{\mathrm{AB}} / f_{\mathrm{AB}}$, where $h_{\mathrm{AB}}$ and $f_{\mathrm{AB}}$ are constants independent of $X(k, t)$. Then, we can write

$$
P_{\mathrm{C}_{6} \mathrm{~B}_{i} \mathrm{~A}_{2 j}}(k, t)=\frac{i !}{j !(i-j) !} \alpha^{j}(1-\alpha)^{i-j} P_{\mathrm{C}_{6} \mathrm{~B}_{i}}(k, t),
$$

with $\alpha=\frac{x_{\mathrm{A}}(t) g_{\mathrm{CB}: \mathrm{A}}}{1+x g_{\mathrm{CB}: \mathrm{A}}}$, and $P_{\mathrm{C}_{6} \mathrm{~B}_{i}}(k, t)=\sum_{j=0}^{i} P_{\mathrm{C}_{6} \mathrm{~B}_{i} \mathrm{~A}_{2 j}}(k, t)$. Using the variables $P_{\mathrm{C}_{6} \mathrm{~B}_{i}}(k, t)$, the kinetic equation for $P_{\mathrm{C}_{6} \mathrm{~B}_{i} \mathrm{~A}_{2 j}}(k, t)$ becomes

$$
\begin{aligned}
\frac{d}{d t} P_{\mathrm{C}_{6} \mathrm{~B}_{i}}(k, t)= & (7-i) h_{\mathrm{B}} x_{\mathrm{B}}(t) P_{\mathrm{C}_{6} \mathrm{~B}_{i-1}}(k, t)-i f_{\mathrm{B}} P_{\mathrm{C}_{6} \mathrm{~B}_{i}}(k, t) \\
& -(6-i) h_{\mathrm{B}} x_{\mathrm{B}}(t) P_{\mathrm{C}_{6} \mathrm{~B}_{i}}(k, t)+(i+1) f_{\mathrm{B}} P_{\mathrm{C}_{6} \mathrm{~B}_{i+1}}(k, t), \quad \text { for } 1 \leq i \leq 5, \\
\frac{d}{d t} P_{\mathrm{C}_{6} \mathrm{~B}_{0}}(k, t)= & -6 h_{\mathrm{B}} x_{\mathrm{B}}(t) P_{\mathrm{C}_{6} \mathrm{~B}_{0}}(k, t)+f_{\mathrm{B}} P_{\mathrm{C}_{6} \mathrm{~B}_{1}}(k, t), \\
\frac{d}{d t} P_{\mathrm{C}_{6} \mathrm{~B}_{6}}(k, t)= & h_{\mathrm{B}} x_{\mathrm{B}}(t) P_{\mathrm{C}_{6} \mathrm{~B}_{5}}(k, t)-6 f_{\mathrm{B}} P_{\mathrm{C}_{6} \mathrm{~B}_{6}}(k, t),
\end{aligned}
$$

where the rate constants are

$$
\begin{aligned}
& h_{\mathrm{B}}(k, t)=h_{\mathrm{B} 0}\left[1-\tanh \left(\frac{2 X(k, t)-1}{B_{X}}\right)\right], \\
& f_{\mathrm{B}}(k, t)=f_{\mathrm{B} 0}\left[1+\tanh \left(\frac{2 X(k, t)-1}{B_{X}}\right)\right] .
\end{aligned}
$$

$h_{\mathrm{B} 0}$, and $f_{\mathrm{B} 0}$ are the rate constants defining the time scale and $B_{X}>0$ is a constant determining the sensitivity to the structure. Because the bindable conformation of KaiB appears with the thermal activation with the energy $\Delta E_{\mathrm{fs}-\mathrm{gs}}$, we assume $h_{\mathrm{B} 0}$ at temperature $T$ is

$$
h_{\mathrm{B} 0}(T)=h_{\mathrm{B} 0}\left(T_{0}\right) \exp \left(-\frac{\Delta E_{0}+\Delta E_{\mathrm{fs}-\mathrm{gs}}}{k_{\mathrm{B}} T}+\frac{\Delta E_{0}+\Delta E_{\mathrm{fs}-\mathrm{gs}}}{k_{\mathrm{B}} T_{0}}\right),
$$

as explained in Table 1 in the main text. 


\section{P/dP kinetics}

For the $\mathrm{P} / \mathrm{dP}$ reactions, we calculate $D(k, t)=\sum_{\mathscr{D}} \mathscr{D} P(\mathscr{D}(k), t)$. Then, we have

$$
\frac{d}{d t} D(k, t)=k_{\mathrm{p}} H^{+}(k, t)[1-D(k, t)]-k_{\mathrm{dp}} H^{-}(k, t) D(k, t),
$$

with the rate constants $k_{\mathrm{p}}$ and $k_{\mathrm{dp}}$. Here, $H^{+}(k, t)=z /(1+z)$ and $H^{-}(k, t)=1 /(1+z)$ are the effects of binding and unbinding of KaiA to and from the CII, respectively, and $z=P_{\mathrm{C}_{6} \mathrm{~A}_{2}}(k, t) / P_{0}$ with a constant $P_{0}$.

\section{ATPase reactions}

We describe the non-equilibrium ATPase reactions by using a stochastic variable $q(i ; k, t)$. When ATP is bound on the $i$ th CI domain of the $k$ th KaiC hexamer, we write $q(i ; k, t)=0$. The ATP hydrolysis is represented by the transition from $q(i ; k, t)=0$ to $q(i ; k, t)=1$, which takes place at a random timing with the frequency $f_{\text {hyd }}$. We approximate that $f_{\text {hyd }}$ does not depend on $X$, as explained in the main text. The state $q(i ; k, t)=1$ represents the ADP-bound state. It is not known how the release of inorganic phosphate $\left(\mathrm{P}_{\mathrm{i}}\right)$ impacts the KaiC structure. In the present study, for simplicity, we do not distinguish the ADP+P bound state just after the hydrolysis and the ADP bound state after the $\mathrm{P}_{\mathrm{i}}$ release. We assume that the lifetime of the ADP bound state $\hat{\Delta}_{\mathrm{ADP}}(k, t)$ is stochastically fluctuating as

$$
\hat{\Delta}_{\mathrm{ADP}}(k, t)=\Delta_{\mathrm{ADP}}(k, t)+\xi(k, t),
$$

where $\xi(k, t)$ is a random number satisfying $\langle\xi(k, t)\rangle=0$ and $\left\langle\xi(k, t) \xi\left(k^{\prime}, t^{\prime}\right)\right\rangle=\delta_{k k^{\prime}} \delta\left(t-t^{\prime}\right) \Delta_{\mathrm{ADP}}(k, t)$. After the ADP release, the ATP rebinds, which turns the nucleotide-binding state from $q(i ; k, t)=1$ to 0 . As explained in the main text, we assume

$$
f_{\text {hyd }}=\text { const. }
$$

and

$$
\Delta_{\mathrm{ADP}}(k, t)=\Delta_{\mathrm{ADP}}^{0}\left[1-\tanh \left(\frac{2 X(k, t)-1}{C_{X}}\right)\right],
$$

where $C_{X}$ and $\Delta_{\mathrm{ADP}}^{0}$ are constants.

The ensemble-averaged rate of the ADP release, $\bar{q}_{r}(t)$, shown in Fig. 2B, was calculated as

$$
\bar{q}_{r}(t)=\frac{1}{\Delta t} \sum_{t \in \Delta t}\left(\frac{1}{6 N} \sum_{k=1}^{N} \sum_{i=1}^{6} \theta[q(i ; k, t-\delta t)-q(i ; k, t)]\right),
$$

where $\theta(x)=1$ for $x>0$ and $\theta(x)=0$ for $x \leq 0 . \delta t=10^{-3} \mathrm{~h}$ is the width of the simulation time step, and $\Delta t=0.2 \mathrm{~h}$ is the time window for the data sampling.

\section{Self-consistent relations}

The constraints coming from the conservation of the total concentrations of KaiA (Eq. 5 of the main text) and KaiB are simplified with the quasi-equilibrium treatment of KaiA (Eqs. 17 and 19) as

$$
\begin{gathered}
x_{\mathrm{A}}(t)+x_{\mathrm{A}}(t)\left[\frac{1}{V} \sum_{k=1}^{N} g_{\mathrm{C}: \mathrm{A}}(k, t) P_{\mathrm{C}_{6} \mathrm{~B}_{0}}(k, t)\right]+\frac{x_{\mathrm{A}}(t) g_{\mathrm{CB}: \mathrm{A}}}{1+x_{\mathrm{A}}(t) g_{\mathrm{CB}: \mathrm{A}}}\left[\frac{1}{V} \sum_{k=1}^{N} \sum_{i=1}^{6} i P_{\mathrm{C}_{6} \mathrm{~B}_{i}}(k, t)\right]=A_{\mathrm{T}} / 2, \\
x_{\mathrm{B}}(t)+\frac{1}{V} \sum_{k=1}^{N} \sum_{i=1}^{6} i P_{\mathrm{C}_{6} \mathrm{~B}_{i}}(k, t)=B_{\mathrm{T}} .
\end{gathered}
$$

$R(k, t)$ in Eq. 13 represents the major assumptions on the feedback coupling in the present model. We use $R(k, t)=d_{0}+$ $d_{1} P_{\mathrm{C}_{6} \mathrm{~A}_{2}}(k, t)-d_{2} \sum_{i=0}^{6} \sum_{j=0}^{i} P_{\mathrm{C}_{6} \mathrm{~B}_{i} \mathrm{~A}_{2 j}}(k, t)-d_{3}[D(k, t)-(1-D(k, t))]-d_{4} F(q(k, t))$ with constants $d_{0}, d_{1}, d_{2}, d_{3}$, and $d_{4}$, and

$$
F(q(k, t))=q(k, t) X(k, t)-(1-q(k, t))(1-X(k, t))
$$

Then, we have

$$
\begin{array}{r}
X(k, t)=\frac{1}{2}\left\{1+\tanh \left[\beta \left(d_{0}+d_{1} P_{\mathrm{C}_{6} \mathrm{~A}_{2}}(k, t)-d_{2} \sum_{i=0}^{6} \sum_{j=0}^{i} P_{\mathrm{C}_{6} \mathrm{~B}_{i} \mathrm{~A}_{2 j}}(k, t)\right.\right.\right. \\
\left.\left.\left.-d_{3}[D(k, t)-(1-D(k, t))]-d_{4} F(q(k, t))\right)\right]\right\} .
\end{array}
$$


Table 2. Rate constants in the model.

\begin{tabular}{llll}
\hline Constants & $\begin{array}{l}\text { Standard } \\
\text { values }\left(\mathrm{h}^{-1}\right)^{*}\end{array}$ & Reactions & Equations \\
\hline$h_{\mathrm{A} 0}$ & $\begin{array}{l}5 \times 10^{-1} \\
f_{\mathrm{A} 0}\end{array}$ & $\begin{array}{l}\text { KaiA binding to KaiC } \\
\text { KaiA unbinding from KaiC }\end{array}$ & $\begin{array}{l}\text { Eq. 18 } \\
\text { Eq. 18 }\end{array}$ \\
\hline$h_{\mathrm{B} 0}$ & $5 \times 10^{-5}$ & KaiB binding to KaiC & Eqs. 22 and 23, \\
$f_{\mathrm{B} 0}$ & 2 & KaiB unbinding from KaiC & Eq. 22 \\
\hline$h_{\mathrm{AB}}$ & $1 \times 10^{-1}$ & KaiA binding to KaiB & Eqs. 19 and 20 \\
$f_{\mathrm{AB}}$ & 1 & KaiA unbinding from KaiB & Eqs. 19 and 20 \\
\hline$k_{\mathrm{p}}$ & 0.22 & Phosphorylation (P) & Eq. 24 \\
$k_{\mathrm{dp}}$ & 0.22 & Dephosphorylation (dP) & Eq. 24 \\
\hline$f_{\text {hyd }}$ & 1 & Hydrolysis freq. of the bound ATP & Eq. 26 \\
$\Delta_{\mathrm{ADP}}^{0}-1$ & 1 & $1 /$ (lifetime) of the bound ADP & Eq. 27 \\
\hline
\end{tabular}

*Values defined at $T=T_{0}$. Concentrations are defined in units of $V=1$.

\section{Simulations}

We simulated the system containing $N=1000$ or 2000 KaiC hexamers by numerically integrating the kinetics with a time step of $\delta t=10^{-3} \mathrm{~h}$. The variables describing the system are $P_{\mathrm{C}_{6} \mathrm{~A}_{2}}(k, t), P_{\mathrm{C}_{6} \mathrm{~B}_{i} \mathrm{~A}_{2 j}}(k, t)(0 \leq j \leq i \leq 6), q(i ; k, t)(1 \leq i \leq 6), D(k, t)$, $X(k, t)$, for $k=1 \sim N, x_{\mathrm{A}}(t)$ and $x_{\mathrm{B}}(t)$. From given values of these variables at time $t$, the values at $t+\delta t$ were obtained by (i) stochastically updating $q(i ; k, t)$ using a constant $f_{\text {hyd }}$ or Eqs. 25 and 27, (ii) evaluating the binding and unbinding constants of Eqs. 18 and 22, (iii) updating $P_{\mathrm{C}_{6} \mathrm{~A}_{2}}(k, t)$ and $P_{\mathrm{C}_{6} \mathrm{~B}_{i} \mathrm{~A}_{2 j}}(k, t)$ with Eqs. 17 and 21, (iv) updating $x_{\mathrm{A}}$ and $x_{\mathrm{B}}$ by solving Eqs. 29 and 30, (v) updating $D(k, t)$ with Eq. 24 , and (vi) updating $X(k, t)$ using Eq. 32. The period and amplitude were calculated from the trajectories, each having the length $3276.8 \mathrm{~h}$ obtained after the initial warming-up trajectories of $100 \mathrm{~h}$ length.

\section{Parameters}

The KaiC concentration is $C_{\mathrm{T}}=3.3 \mu \mathrm{M}$ on a monomer basis for $N=1000$ and $V=3 \times 10^{-15} l$; this concentration is close to $3.5 \mu \mathrm{M}$, often used in experiments. We assume the ratio $A_{\mathrm{T}}: B_{\mathrm{T}}: C_{\mathrm{T}}=1: 3: 3$ as in many experiments ${ }^{23,54,67}$. The oscillations were robust against small parameter changes; therefore, we did not calibrate the parameters but determined them from the order of magnitude argument. We chose $h_{\mathrm{B} 0} B_{\mathrm{T}}$ and $f_{\mathrm{B} 0}$ to satisfy $h_{\mathrm{B} 0} B_{\mathrm{T}} \approx f_{\mathrm{B} 0} \approx 1 \mathrm{~h}^{-1}$. We used $h_{\mathrm{B} 0}=5 \times 10^{-5} \mathrm{~h}^{-1}$ and $f_{\mathrm{B} 0}=2 \mathrm{~h}^{-1}$ in units of $V=1$, corresponding to the dissociation constant of $K_{d}^{\mathrm{C}: \mathrm{B}}=2.2 \times 10 \mu \mathrm{M}$ in $V=3 \times 10^{-15} l$. We used $h_{\mathrm{A} 0} / f_{\mathrm{A} 0}=5 \times 10^{-4}$ in units of $V=1$, corresponding to $K_{d}^{\mathrm{C}: \mathrm{A}}=1.1 \mu \mathrm{M}$ in $V=3 \times 10^{-15} l$, which agrees with the experimentally observed values of $K_{d}^{\mathrm{C}: \mathrm{A}} \sim \mu \mathrm{M}^{72}$. The dissociation constant of KaiA and KaB was not yet observed experimentally. Here, we assumed a rather small value to ensure the sequestration effect; $g_{\mathrm{CB}: \mathrm{A}}=1 \times 10^{-1}$ in units of $V=1$, corresponding to $K_{d}^{\mathrm{CB}: \mathrm{A}}=5.56 \mathrm{nM}$ in $V=3 \times 10^{-15} l$. See Tables 2 and 3 for values of the other parameters. We chose $d_{0}, d_{1}$, $d_{2}, d_{3}$. and $d_{4}$ as of the order of $k_{\mathrm{B}} T_{0}$, and $A_{X}, B_{X}$, and $C_{X}$ as of the order of one.

\section{Acknowledgments}

This work was supported by JSPS-KAKENHI Grants JP19H01860, 19H05258 and 20H05530. 
Table 3. Other parameters.

\begin{tabular}{llll}
\hline Constants & $\begin{array}{l}\text { Standard } \\
\text { values }\end{array}$ & Implications & Equations \\
\hline$N$ & 1000 or 2000 & \# of KaiC hexamers in the simulation & Eqs. 29 and 30 \\
$\frac{B_{\mathrm{T}}}{C_{\mathrm{T}} / 6}$ & $6 N$ & \# of KaiB monomers in the simulation & Eqs. 29 and 30 \\
$\frac{A_{\mathrm{T}} / 2}{C_{\mathrm{T}} / 6}$ & $N$ & \# of KaiA dimers in the simulation & Eqs. 29 and 30 \\
$V$ & 1 & Volume in the simulation & Eqs. 29 and 30 \\
$T_{0}$ & $30{ }^{\circ} \mathrm{C}$ & Standard temperature & \\
$\delta t$ & $10^{-3} \mathrm{~h}$ & Width of the simulation time step & \\
\hline$d_{0}$ & $2 k_{\mathrm{B}} T_{0}$ & Determining the average structure & Eq. 32 \\
$d_{1}$ & $5 k_{\mathrm{B}} T_{0}$ & Coupling strength of struct.-KaiA bind/unbind & Eq. 32 \\
$d_{2}$ & $5 k_{\mathrm{B}} T_{0}$ & Coupling strength of struct.-KaiB bind/unbind & Eq. 32 \\
$d_{3}$ & $3 k_{\mathrm{B}} T_{0}$ & Coupling strength of struct.-P/dP reactions & Eq. 32 \\
$d_{4}$ & $2 k_{\mathrm{B}} T_{0}$ & Coupling strength of struct.-ATPase reactions & Eq. 32 \\
\hline$A_{X}$ & 1 & Sensitivity of KaiA binding to KaiC structure & Eq. 18 \\
$B_{X}$ & 1 & Sensitivity of KaiB binding to KaiC structure & Eq. 22 \\
$C_{X}$ & 2 & Sensitivity of ADP lifetime to KaiC structure & Eq. 27 \\
$P_{0}$ & $1 \times 10^{-1}$ & Sensitivity of P/dP reactions to KaiA binding & Eq. 24 \\
\hline
\end{tabular}




\section{References}

1. Nakajima, M. et al. Reconstitution of circadian oscillation of cyanobacterial KaiC phosphorylation in vitro. Science $\mathbf{3 0 8}$, 414-415, DOI: https://doi.org/10.1126/science.1108451 (2005).

2. Akiyama, S. Structural and dynamic aspects of protein clocks: how can they be so slow and stable? Cell Mol Life Sci 69, 2147-2160, DOI: https://doi.org/10.1007/s00018-012-0919-3 (2012).

3. Egli, M. Intricate protein-protein interactions in the cyanobacterial circadian clock. J Biol Chem 289, 21267-21275, DOI: https://doi.org/10.1074/jbc.R114.579607 (2014).

4. Dunlap, J. C. Molecular bases for circadian clocks. Cell 96, 271-290, DOI: https://doi.org/10.1016/s0092-8674(00)80566-8 (1999).

5. Bell-Pedersen, D. et al. Circadian rhythms from multiple oscillators: lessons from diverse organisms. Nat Rev Genet. 6 , 544-556, DOI: https://doi.org/10.1038/nrg1633 (2005).

6. Pittendrigh, C. S. On temperature independence in the clock system controlling emergence time in Drosophila. Proc. Natl. Acad. Sci. USA 40, 1018-1029, DOI: https://doi.org/10.1073/pnas.40.10.1018 (1954).

7. Hastings, J. W. \& Sweeney, B. M. On the mechanism of temperature independence in a biological clock. Proc. Natl. Acad. Sci. USA 43, 804-811, DOI: https://doi.org/10.1073/pnas.43.9.804 (1957).

8. Lakin-Thomas, P., Brody, S. \& Coté, G. G. Amplitude model for the effects of mutations and temperature on period and phase resetting of the Neurospora circadian oscillator. J. Biol. Rhythm. 6, 281-297, DOI: https://doi.org/10.1177/ 074873049100600401 (1991).

9. Ruoff, P. Introducing temperature-compensation in any reaction kinetic oscillator model. J. Interdiscipl. Cycle Res. 23, 92-99, DOI: https://doi.org/10.1080/09291019209360133 (1992).

10. Ruoff, P., Loros, J. J. \& Dunlap, J. C. The relationship between FRQ-protein stability and temperature compensation in the Neurospora circadian clock. Proc. Natl. Acad. Sci. USA 102, 17681-17686, DOI: https://doi.org/10.1073/pnas.0505137102 (2005).

11. Kurosawa, G. \& Iwasa, Y. Temperature compensation in circadian clock models. J. Theor. Biol. 233, 453-468, DOI: https://doi.org/10.1016/j.jtbi.2004.10.012 (2005).

12. Hong, C. I., Conrad, E. D. \& John J. Tyson, J. T. A proposal for robust temperature compensation of circadian rhythms. Proc. Natl. Acad. Sci. USA 104, 1195-1200, DOI: https://doi.org/10.1073/pnas.0601378104 (2007).

13. Leloup, J.-C. \& Albert Goldbeter, A. Temperature compensation of circadian rhythms: Control of the period in a model for circadian oscillations of the per protein in Drosophila. Chronobiol. Intern. 14, 511-520, DOI: https://doi.org/10.3109/ 07420529709001472 (2009).

14. Bodenstein, C., Heiland, I. \& Schuster, S. Temperature compensation and entrainment in circadian rhythms. Physi. Biol. 9, 036011, DOI: https://doi.org/10.1088/1478-3975/9/3/036011 (2012).

15. François, P., Despierre, N. \& Siggia, E. D. Adaptive temperature compensation in circadian oscillations. PLoS Comp. Biol. 8, e1002585, DOI: https://doi.org/doi:10.1371/journal.pcbi.1002585 (2012).

16. Kidd, P. B., Young, M. W. \& Siggia, E. D. Temperature compensation and temperature sensation in the circadian clock. Proc. Natl. Acad. Sci. USA 112, E6284-E6292, DOI: https://doi.org/10.1073/pnas.1511215112 (2015).

17. Kurosawa, G., Fujioka, A., Koinuma, S., Mochizuki, A. \& Shigeyoshi, Y. Temperature-amplitude coupling for stable biological rhythms at different temperatures. PLoS Comp. Biol. 13, e1005501, DOI: https://doi.org/10.1371/journal.pcbi. 1005501 (2017).

18. Avello, P., .Davis, S. J., Ronald, J. \& .Pitchford, J. W. Heat the clock: entrainment and compensation in Arabidopsis circadian rhythms. J. Circadian Rhythm. 17, 1-11, DOI: https://doi.org/10.5334/jcr.179 (2019).

19. Avello, P., .Davis, S. J. \& .Pitchford, J. W. Temperature robustness in Arabidopsis circadian clock models is facilitated by repressive interactions, autoregulation, and three-node feedbacks. J Theor. Biol. 509, 110495, DOI: https://doi.org/10.1016/ j.jtbi.2020.110495 (2021).

20. Ruoff, P., Rensing, L., Kommedal, R. \& Mohsenzadeh, S. Modeling temperature compensation in chemical and biological oscillators. Chronobiol Int. 14, 499-510, DOI: https://doi.org/10.3109/07420529709001471 (1997).

21. Isojima, Y. et al. CKI $\varepsilon / \delta$-dependent phosphorylation is a temperature-insensitive, period-determining process in the mammalian circadian clock. Proc. Natl. Acad. Sci. USA 106, 15744-15749, DOI: https://doi.org/10.1073/pnas.0908733106 (2009). 
22. Shinohara, Y. et al. Temperature-sensitive substrate and product binding underlie temperature-compensated phosphorylation in the clock. Mol. Cell 67, 783-798, DOI: https://doi.org/10.1016/j.molcel.2017.08.009 (2017).

23. Terauchi, K. et al. ATPase activity of KaiC determines the basic timing for circadian clock of cyanobacteria. Proc Natl Acad Sci U S A 104, 16377-16381, DOI: https://doi.org/10.1073/pnas.0706292104 (2007).

24. Abe, J. et al. Circadian rhythms. atomic-scale origins of slowness in the cyanobacterial circadian clock. Science 349, 312-316, DOI: https://doi.org/10.1126/science.1261040 (2015).

25. Hatakeyama, T. S. \& Kaneko, K. Generic temperature compensation of biological clocks by autonomous regulation of catalyst concentration. Proc Natl Acad Sci U S A 109, 8109-8114, DOI: https://doi.org/10.1073/pnas.1120711109 (2012).

26. Ito-Miwa, K., Furuike, Y., Akiyama, S. \& Kondo, T. Tuning the circadian period of cyanobacteria up to 6.6 days by the single amino acid substitutions in kaic. Proc. Natl. Acad. Sci. USA 117, 20926-20931, DOI: https://doi.org/10.1073/pnas. 2005496117 (2020).

27. Furuike, Y. et al. Elucidation of master allostery essential for circadian clock oscillation in cyanobacteria. bioRxiv doi.org/10.1101/2021.08.30.457330, DOI: https://doi.org/10.1101/2021.08.30.457330 (2021).

28. Emberly, E. \& Wingreen, N. S. Hourglass model for a proteinbased circadian oscillator. Phys Rev Lett 96, 038303, DOI: https://doi.org/10.1103/PhysRevLett.96.038303 (2006).

29. Mori, T. et al. Elucidating the ticking of an in vitro circadian clockwork. PLoS Biol 5, e93, DOI: https://doi.org/10.1371/ journal.pbio.0050093 (2007).

30. Yoda, M., Eguchi, K., Terada, T. P. \& Sasai, M. Monomer-shuffling and allosteric transition in KaiC circadian oscillation. PLoS One 2, e408, DOI: https://doi.org/10.1371/journal.pone.0000408 (2007).

31. Eguchi, K., Yoda, M., Terada, T. P. \& Sasai, M. Mechanism of robust circadian oscillation of KaiC phosphorylation in vitro. Biophys J 95, 1773-1784, DOI: https://doi.org/10.1529/biophysj.107.127555 (2008).

32. Nagai, T., Terada, T. P. \& Sasai, M. Synchronization of circadian oscillation of phosphorylation level of KaiC in vitro. Biophys J 98, 2469-2477, DOI: https://doi.org/10.1016/j.bpj.2010.02.036 (2010).

33. Zhang, D., Cao, Y., Ouyang, Q. \& Tu, Y. The energy cost and optimal design for synchronization of coupled molecular oscillators. Nat. Phys. 16, 95-100, DOI: https://doi.org/10.1038/s41567-019-0701-7 (2019).

34. Takigawa-Imamura, H. \& Mochizuki, A. Predicting regulation of the phosphorylation cycle of KaiC clock protein using mathematical analysis. J Biol Rhythm. 21, 405-416, DOI: https://doi.org/10.1177/0748730406291329 (2006).

35. van Zon, J. S., Lubensky, D. K., Altena, P. R. \& ten Wolde, P. R. An allosteric model of circadian KaiC phosphorylation. Proc Natl Acad Sci U S A 104, 7420-7425, DOI: https://doi.org/10.1073/pnas.0608665104 (2007).

36. Mori, T. et al. Revealing circadian mechanisms of integration and resilience by visualizing clock proteins working in real time. Nat Commun 9, 3245, DOI: https://doi.org/10.1038/s41467-018-05438-4 (2018).

37. Rust, M. J., Markson, J. S., Lane, W. S., Fisher, D. S. \& O'Shea, E. K. Ordered phosphorylation governs oscillation of a three-protein circadian clock. Science 318, 809-812, DOI: https://doi.org/10.1126/science.1148596 (2007).

38. Wang, J., Xu, L. \& Wang, E. Robustness and coherence of a three-protein circadian oscillator: Landscape and flux perspectives. Biophys J 97, 3038-3046, DOI: https://doi.org/10.1016/j.bpj.2009.09.021 (2009).

39. Phong, C., Markson, J. S., Wilhoite, C. M. \& Rust, M. J. Robust and tunable circadian rhythms from differentially sensitive catalytic domains. Proc Natl Acad Sci U S A 110, 1124-1129, DOI: https://doi.org/10.1073/pnas.1212113110 (2013).

40. Paijmans, J., Lubensky, D. K. \& ten Wolde, P. R. A thermodynamically consistent model of the post-translational Kai circadian clock. PLoS Comput. Biol 13, e1005415, DOI: https://doi.org/10.1371/journal.pcbi.1005415 (2017).

41. Das, S., Terada, T. P. \& Sasai, M. Role of ATP hydrolysis in cyanobacterial circadian oscillator. Sci Rep 7, 17469, DOI: https://doi.org/10.1038/s41598-017-17717-z (2017).

42. Das, S., Terada, T. P. \& Sasai, M. Single-molecular and ensemble-level oscillations of cyanobacterial circadian clock. Biophys Physicobiol 15, 136-150, DOI: https://doi.org/10.2142/biophysico.15.0_136 (2018).

43. Sasai, M. Effects of stochastic single-molecule reactions on coherent ensemble oscillations in the KaiABC circadian clock. J Phys Chem B 123, 702-713, DOI: https://doi.org/10.1021/acs.jpcb.8b10584 (2019).

44. Sasai, M. Mechanism of autonomous synchronization of the circadian KaiABC rhythm. Sci. Rep. 11, 4713, DOI: https://doi.org/10.1038/s41598-021-84008-z (2021). 
45. Nakajima, M., Ito, H. \& Kondo, T. In vitro regulation of circadian phosphorylation rhythm of cyanobacterial clock protein KaiC by KaiA and KaiB. FEBS Lett 584, 898-902, DOI: https://doi.org/10.1016/j.febslet.2010.01.016 (2010).

46. Ito, H. et al. Autonomous synchronization of the circadian KaiC phosphorylation rhythm. Nat Struct Mol Biol 14, 1084-1088, DOI: https://doi.org/10.1038/nsmb1312 (2007).

47. Mori, T. et al. Circadian clock protein KaiC forms ATP-dependent hexameric rings and binds DNA. Proc Natl Acad Sci U S A 99, 17203-17208, DOI: https://doi.org/10.1073/pnas.262578499 (2002).

48. Hayashi, F. et al. ATP-induced hexameric ring structure of the cyanobacterial circadian clock protein KaiC. Genes to Cells 8, 287-296, DOI: https://doi.org/10.1046/j.1365-2443.2003.00633.x (2003).

49. Pattanayek, R. et al. Visualizing a circadian clock protein: crystal structure of KaiC and functional insights. Mol Cell 15, 375-388, DOI: https://doi.org/10.1016/j.molcel.2004.07.013 (2004).

50. Iwasaki, H., Taniguchi, Y., Ishiura, M. \& Kondo, T. Physical interactions among circadian clock proteins kaia, kaib and kaic in cyanobacteria. EMBO J 18, 1137-1145, DOI: https://doi.org/10.1093/emboj/18.5.1137 (1999).

51. Chang, Y. G., Kuo, N. W., Tseng, R. \& LiWang. Flexibility of the C-terminal, or CII, ring of KaiC governs the rhythm of the circadian clock of cyanobacteria. Proc Natl Acad Sci U S A 108, 14431-14436, DOI: https://doi.org/10.1073/pnas. 1104221108 (2011).

52. Chang, Y. G., Tseng, R., Kuo, N. W. \& LiWang, A. Rhythmic ring-ring stacking drives the circadian oscillator clockwise. Proc Natl Acad Sci U S A 109, 16847-16851, DOI: https://doi.org/10.1073/pnas.1211508109 (2012).

53. Murayama, Y. et al. Tracking and visualizing the circadian ticking of the cyanobacterial clock protein KaiC in solution. EMBO J 30, 68-78, DOI: https://doi.org/10.1038/emboj.2010.298 (2011).

54. Oyama, K., Azai, C., Nakamura, K., Tanaka, S. \& Terauchi, K. Conversion between two conformational states of KaiC is induced by ATP hydrolysis as a trigger for cyanobacterial circadian oscillation. Sci Rep 6, 32443, DOI: https: //doi.org/10.1038/srep32443 (2016).

55. Iwasaki, H., Nishiwaki, T., Kitayama, Y., Nakajima, M. \& Kondo, T. KaiA-stimulated KaiC phosphorylation in circadian timing loops in cyanobacteria. Proc Natl Acad Sci U S A 99, 15788-15793, DOI: https://doi.org/10.1073/pnas.222467299 (2002).

56. Pattanayek, R. et al. Analysis of KaiA-KaiC protein interactions in the cyano-bacterial circadian clock using hybrid structural methods. EMBO J 25, 2017-2028, DOI: https://doi.org/10.1038/sj.emboj.7601086 (2006).

57. Kitayama, Y., Iwasaki, H., Nishiwaki, T. \& Kondo, T. KaiB functions as an attenuator of KaiC phosphorylation in the cyanobacterial circadian clock system. EMBO J 22, 2127-2134, DOI: https://doi.org/10.1093/emboj/cdg212 (2003).

58. Xu, Y., Mori, T. \& Johnson, C. H. Cyanobacterial circadian clockwork: roles of KaiA, KaiB and the KaiBC promoter in regulating KaiC. EMBO J 22, 2117-2126, DOI: https://doi.org/10.1093/emboj/cdg168 (2003).

59. Kitayama, Y., Nishiwaki-Ohkawa, T., Sugisawa, Y. \& Kondo, T. KaiC intersubunit communication facilitates robustness of circadian rhythms in cyanobacteria. Nat Commun 4, 2897, DOI: https://doi.org/10.1038/ncomms3897 (2013).

60. Snijder, J. et al. Structures of the cyanobacterial circadian oscillator frozen in a fully assembled state. Science 355, 1181-1184, DOI: https://doi.org/10.1126/science.aag3218 (2017).

61. Pattanayek, R. \& Egli, M. Protein-protein interactions in the cyanobacterial circadian clock: structure of KaiA dimer in complex with C-terminal KaiC peptides at 2.8 å resolution. Biochemistry 54, 4575-4578, DOI: https://doi.org/10.1021/acs. biochem.5b00694 (2015).

62. Chang, Y.-G. et al. A protein fold switch joins the circadian oscillator to clock output in cyanobacteria. Science 17, 324-328, DOI: https://doi.org/10.1126/science.1260031 (2015).

63. Henzler-Wildman, K. \& Kern, D. Dynamic personalities of proteins. Nature 450, 964-972, DOI: https://doi.org/10.1038/ nature06522 (2007).

64. Mutoh, R., Nishimura, A., Yasui, S., Onai, K. \& Ishiura, M. The ATP-mediated regulation of KaiB-KaiC interaction in the cyanobacterial circadian clock. PLoS One 8, e80200, DOI: https://doi.org/10.1371/journal.pone.0080200 (2013).

65. Mukaiyama, A. et al. Conformational rearrangements of the $\mathrm{C} 1$ ring in KaiC measure the timing of assembly with KaiB. Sci Rep 8, 8803, DOI: https://doi.org/10.1038/s41598-018-27131-8 (2018).

66. Tseng, R. et al. Structural basis of the day-night transition in a bacterial circadian clock. Science 355, 1174-1180, DOI: https://doi.org/10.1126/science.aag2516 (2017). 
67. Nishiwaki, T. et al. A sequential program of dual phosphorylation of $\mathrm{KaiC}$ as a basis for circadian rhythm in cyanobacteria. EMBO J 26, 4029-4037, DOI: https://doi.org/10.1038/sj.emboj.7601832 (2007).

68. Furuike, Y. et al. Cross-scale analysis of temperature compensation in the cyanobacterial circadian clock system. bioRxiv doi.org/10.1101/2021.08.20.457041, DOI: https://doi.org/10.1101/2021.08.20.457041 (2021).

69. Murayama, Y. et al. Low temperature nullifies the circadian clock in cyanobacteria through Hopf bifurcation. Proc Natl Acad Sci U S A 114, 5641-5646, DOI: https://doi.org/10.1073/pnas.1620378114 (2017).

70. Sasai, M. \& Wolynes, P. G. Stochastic gene expression as a manybody problem. Proc. Natl. Acad. Sci. U S A 100, 2374-2379, DOI: https://doi.org/10.1073/pnas.2627987100 (2003).

71. Walczak, A. M., Sasai, M. \& Wolynes, P. G. Self-consistent proteomic field theory of stochastic gene switches. Biophys $J$ 88, 828-850, DOI: https://doi.org/10.1529/biophysj.104.050666 (2005).

72. Kageyama, H. et al. Cyanobacterial circadian pacemaker: Kai protein complex dynamics in the KaiC phosphorylation cycle in vitro. Mol Cell 23, 161-171, DOI: https://doi.org/10.1016/j.molcel.2006.05.039 (2006). 